\title{
Exact Controllability of a Pluridimensional Coupled Problem
}

\author{
Serge NiCAISE
}

ABSTRACT. We set a coupled boundary value problem between two domains of different dimension. The first one is the unit cube of $\mathbf{R}^{n}, n \in\{2,3\}$, with a crack and the second one is the crack. This problem comes from [1], that obtained an analogous coupled problem. We show that the solution has singularities due to the crack. As in [7], we adapt the Hilbert uniqueness method of J.-L. Lions [11-12] in order to obtain the exact controllability of the associated wave equation with Dirichlet action on a part of the boundary.

\section{INTRODUCTION}

Let us start with some notations. For $n \in\{2,3\}$, we set

$$
\begin{aligned}
& \Gamma=\{x \in]-1,1\left[{ }^{n}: x_{2}=0,0<x_{1}<1\right\}, \\
& \omega=\left\{x \in \mathbf{R}^{n}: x_{2}=0,0<x_{1}<2 \text { and if } n=3,-1<x_{3}<1\right\}, \\
& \Omega=]-1,1\left[{ }^{n} \backslash \vec{\Gamma} .\right.
\end{aligned}
$$

We sometimes identify $\Gamma$ and $\omega$ with the open sets $] 0,1[x]-1,1\left[{ }^{n-2}\right.$ and ] $0,2[\times]-1,1\left[{ }^{n-2}\right.$ of $\mathbf{R}^{n-1}$. We also remark that $\Omega$ is the unit cube with a slit along the half-hyperplane $x_{2}=0, x_{1} \geq 0$ (see figures 1 and 2 ).

We denote by $\gamma_{+} u, \gamma_{+} \frac{\partial u}{\partial \nu^{+}}$(resp. $\gamma_{-} u, \gamma_{-} \frac{\partial u}{\partial \nu^{-}}$) the trace of the function $u$ and the trace of the outward normal derivative of $u$ on $\Gamma$ from above (resp. from below) in $\Omega$. $\partial \Omega$ (resp. $\partial \Gamma, \partial \omega$ ) denotes the boundary of $\Omega$ (resp. $\Gamma, \omega)$, and we set $\Gamma_{1}=\partial \Omega \backslash \vec{\Gamma}$. Moreover, in the sequel, for a function $u$ defined

1991 Mathemnatics Subject Classification: 35C20, 49A22, 49E15.

Editorial Complutense. Madrid, 1992. 


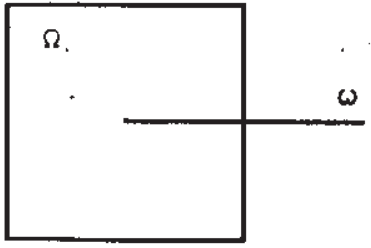

Fig. I $(n=2)$

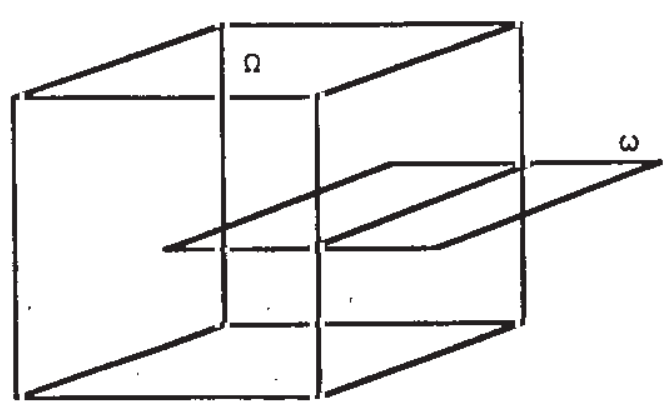

Fig. $2(n=3)$

in $\Omega$ we shall also denote by $\gamma u$ (resp. $\gamma \frac{\partial u}{\partial \nu}$ ) the trace of $u$ (resp. the trace of the outward normal derivative of $u$ ) on $\Gamma_{1}$. And in order to avoid confusions, for a function $v$ defined in $\Gamma$, we denote by $\gamma^{\#} v$ (resp. $\gamma^{\#} \frac{\partial v}{\partial \nu^{\sharp}}$ ) the trace of $v$ (resp. the trace of the outward normal derivative of $v$ ) on $\partial \Gamma$. Finally, $\nu$ (resp. $\nu^{\sharp}$ ) will be in $\Omega$ (resp. $\Gamma$ ), the unitary outer normal vector on $\Gamma_{1}$ (resp. on $\partial \Gamma$ ).

From now on, $\Delta_{n}$ (resp. $\nabla_{n}$ ) will denote the Laplace operator (resp. the gradient) in $\mathbf{R}^{n}$.

We first consider the following boundary value problem: given $f \in L^{2}(\Omega)$, $g \in L^{2}(\omega)$, find weak solutions $u \in H^{\prime}(\Omega)$ and $u_{1} \in H^{1}(\omega)$ of $(1.1),(1.2)$ and (1.3) hereafter.

$$
\begin{cases}-\Delta_{n} u=f & \text { in } \Omega, \\ -\Delta_{n-1} u_{l}+\gamma_{+} & \frac{\partial u}{\partial \bar{\nu}^{+}}+\gamma_{-} \frac{\partial u}{\partial \bar{\nu}^{-}}=g \\ -\Delta_{n-1} u_{l}=g & \text { in } \omega \backslash \vec{\Gamma} .\end{cases}
$$




$$
\begin{aligned}
& \gamma_{+} u=\gamma_{-} u=u_{1} \text { on } \Gamma \text {. } \\
& \left\{\begin{array}{lll}
u=0 & \text { on } \Gamma_{1}, \\
u_{1}=0 & \text { on } & \partial \Gamma .
\end{array}\right.
\end{aligned}
$$

Our motivation to study problem (1.1)-(1.3) comes from [1], who obtained an analogous coupled problem between $\Omega$ and $\omega$ but more complicated. Actually, they deduced a coupling between the linear elasticity system in $\Omega$ and the plate equation in $\omega$ (in dimension 3 ). This paper is the first step in the study of singularities and exact controllability of the problem given in [1].

Due to the Dirichlet boundary conditions imposed on $\Gamma_{1}$, the boundary value problem (1.1) - (1.3) splits into two problems: the first one is a coupling between $\Omega$ and $\Gamma$ i.e. $u$ is a solution of

$$
\left\{\begin{array}{l}
-\Delta_{n} u=f \quad \text { in } \Omega, \\
-\Delta_{n-1}\left(\gamma_{+} u\right)+\gamma_{+} \frac{\partial u}{\partial \nu^{+}}+\gamma_{-} \frac{\partial u}{\partial \nu^{-}}=g \text { on } \Gamma \\
\gamma_{+} u=\gamma_{-} u \text { on } \Gamma \\
u=0 \text { on } \Gamma_{1}, \\
\gamma_{+} u=0 \text { on } \partial \Gamma .
\end{array}\right.
$$

The second one is the Dirichlet problem in $\omega \backslash \vec{\Gamma}$ i.e.

$$
\left\{\begin{array}{l}
-\Delta_{n-1} u_{1}=g \text { in } \omega \backslash \vec{\Gamma}, \\
u_{1}=0 \text { on } \partial(\omega \backslash \vec{\Gamma}) .
\end{array}\right.
$$

Since this second problem is a Dirichlet problem in a convex domain of $\mathbf{R}^{n-1}$, we never speak about it (for the regularity of the solution, see [5] and for the exact controllability of the associated wave equation, see [12]).

We can say that problem (1.4) is a mixed problem Dirichlet-Ventcel (since on a part of the boundary of $\Omega$, the boundary condition is of Dirichlet type; while on the remainder of the boundary, the boundary condition is of Ventcel type). In dimension 2, Ventcel problems on polygonal domains without slit were studied by $K$, Lemrabet [8-9]. Moreover, the regularity results we obtained in dimension 2 are similar to those of [8].

In view of the exact controllability of the wave equation associated with problem (1.4), we need a regularity result about the solution $u$ of (1.4). 
Classically, the optimal regularity we expect is $H^{2}(\Omega)$ for $u$ and $H^{2}(\Gamma)$ for $\gamma_{+} u$. But, even for $u$, it is clear that it can not have the optimal regularity since it is a solution of a non-homogeneous Dirichlet problem in $\Omega$, which is an open set with a slit (cf. [5] and [7]). Inspired from these results, we can show that $u$ and $\gamma_{+} u$ admit the following expansion:

$$
\begin{aligned}
& u=u_{0}+c \eta(r) r^{1 / 2} \sin \left(\frac{\theta}{2}\right), \\
& \gamma_{+} u=u_{20}-\frac{4}{3} c \eta(r) r^{3 / 2},
\end{aligned}
$$

where $u_{0} \in H^{2}(\Omega) ; u_{20} \in H^{2}(\Gamma) ;(r, \theta)$ denotes polar coordinates with origin $(0,0)$ in the $x_{1}, x_{2}$-plane i.e. $x_{1}+i x_{2}=r e^{i \theta}$, such that the half-line $\theta=0$ is equal to the half-line $x_{2}=0, x_{1}>0$ (this means that the half-hyperplane $\theta=0$ contains the crack $\Gamma) ; \eta \in \mathscr{Q}(\mathbf{R})$ is a cut-off function satisfying $\eta=1$ in a neighbourhood of 0 and $\operatorname{supp} \eta \subset]-\frac{1}{2}, \frac{1}{2}[$; finally, in dimension $2, c$ is a constant and in dimension $3, c=c\left(r, x_{3}\right)$ is a function of the variables $r$ and $x_{3}$ such that $c \in H^{1}(] 0,1[x]-1,1[$ ). The expansion (1.6) shows that $u$ has a singularity due to the crack (in dimension 2 , this is a vertex singularity, while in dimension 3, it is an edge singularity). Moreover (1.6) will induce a singularity to $\gamma_{+} u$ due to the jump of the normal derivative of the singular part $c r^{1 / 2} \sin \left(\frac{\theta}{2}\right)$ of $u$. This singularity of $\gamma_{+} u$ is concentrated at the bottom of the crack $\Gamma_{0}^{2}$, since $c$ is regular far from $\Gamma_{0}$.

Let us now pass to the wave equation. For Cauchy data $\left(\varphi_{0}, \varphi_{00}\right)$ and $\left(\varphi_{1}, \varphi_{01}\right)\left(\varphi_{0}\right.$ and $\varphi_{1}$ are functions defined in $\Omega$, while $\varphi_{00}$ and $\varphi_{01}$ are functions defined in $\Gamma$ ) and a Dirichlet datum $\left(v, v_{0}\right)$ (as above, $v$ is defined on $\partial \Omega \times(0, T)$, while $v_{0}$ is defined on $\partial \Gamma \times(0, T)$; we consider the solution $\varphi$ of the following wave equation:

$$
\left\{\begin{array}{l}
\varphi^{\prime \prime}-\Delta_{n} \varphi=0 \text { in } \Omega \times(0, T) \\
\left(\gamma_{+} \varphi\right)^{\prime \prime}-\Delta_{n-1}\left(\gamma_{+} \varphi\right)+\gamma_{+} \frac{\partial \varphi}{\partial \nu^{+}}+\gamma_{-} \frac{\partial \varphi}{\partial \nu^{-}}=0 \text { in } \Gamma \times(0, T) \\
\gamma_{+} \varphi=\gamma_{-} \varphi \text { on } \Gamma \\
\varphi(0)=\varphi_{0},\left(\gamma_{+} \varphi\right)(0)=\varphi_{00} \\
\varphi^{\prime}(0)=\varphi_{1},\left(\gamma_{+} \varphi\right)^{\prime}(0)=\varphi_{01} \\
\varphi=v \text { on } \Gamma_{1} \times(0, T) \\
\gamma_{+} \varphi=v_{0} \text { on } \partial \Gamma \times(0, T)
\end{array}\right.
$$

where $\varphi^{\prime}$ denotes the partial derivative of $\varphi$ with respect to $t$ i.e. $\varphi^{\prime}=D, \varphi$. 
The problem of the exact controllability consists in finding a time $T$ such that for all Cauchy data, there exists (at least) one Dirichlet datum $\left(u, v_{0}\right)$ such that

$$
\begin{gathered}
\varphi(T)=\varphi^{\prime}(T)=0, \\
(\gamma+\varphi)(T)=(\gamma+\varphi)^{\prime}(T)=0 .
\end{gathered}
$$

So, we have to drive our coupled system to rest, by acting on the boundaries $\partial \Omega$ and $\partial \Gamma$.

We adapt the Hilbert uniqueness method of J.-L. Lions [11-12] in order to apply it to our coupled problem (1.8). Our technics are analogous to those of P. Grisvard [7], who studied the exact controllability of the wave equation in nonsmooth domains (see also [14], for the exact controllability of the vibrating plates equation in a polygon).

In our case, the Hilbert uniqueness method is based on the estimate of the energy of the solution $\varphi$ of (1.8) with homogeneous Dirichlet data (i.e. $v=0$, $v_{0}=0$ ) by the $L^{2}$-norm of the normal derivatives $\gamma \frac{\partial \varphi}{\partial \nu}$ and $\gamma^{\sharp} \frac{\partial(\gamma+\varphi)}{\partial \nu^{\sharp}-}$ of $\varphi$ and $\gamma_{+} \varphi$. This estimate is itself based on the following identity, where $m$ is a vector field, called multiplier of the form $m(x)=x-x_{0}$, where $x_{0}=\left(x_{01}, \ldots, x_{0_{n}}\right)$ satisfies $x_{02}=0$ :

$$
\begin{aligned}
& \int_{\Omega}\left(-\Delta_{n} \varphi\right) m \cdot \nabla_{n} \varphi d x \\
+ & \int_{\Gamma}\left(-\Delta_{n-1}\left(\gamma_{+} \varphi\right)+\gamma_{+} \frac{\partial \varphi}{\partial \nu^{+}}+\gamma_{-} \frac{\partial \varphi}{\partial \nu^{-}}\right) \gamma_{+} m \cdot \nabla_{n-1}\left(\gamma_{+} \varphi\right) d x^{\#} \\
= & \left(1-\frac{n}{2}\right) \int_{\Omega}\left|\nabla_{n} \varphi\right|^{2} d x+\left(1-\frac{n-1}{2}\right) \int_{\Gamma}\left|\nabla_{n-1}\left(\gamma_{+} \varphi\right)\right|^{2} d x^{\sharp} \\
& -\frac{1}{2} \int_{\Gamma_{1}} m \cdot \nu\left(\gamma \frac{\partial \varphi}{\partial \nu}\right)^{2} d \sigma-\frac{1}{2} \int_{\partial \Gamma} \gamma_{+} m \cdot \nu^{\sharp}\left(\gamma^{\sharp} \frac{\partial\left(\gamma_{+} \varphi\right)}{\partial \nu^{\sharp}}\right)^{2} d \sigma^{\sharp} \\
+ & \pi x_{01} \int_{\Gamma_{0}} c^{2}\left(\sigma^{\sharp}\right) d \sigma^{\sharp} / 4,
\end{aligned}
$$

where $\Gamma_{0}=\left\{x \in \partial \Gamma: x_{1}=0\right\}$ is the bottom of the crack and $c$ is the coefficient of the singularity appearing in the decomposition (1.6) of $\varphi$.

The choice of $x_{0}$ is inspired from the results of $\S 7$ of [7]. Geometrically, this means that $x_{0}$ is in the direction of the crack in dimension 2 and in the plane of the crack in dimension 3. 
From (1.9), we can deduce that if $x_{01} \geq 0$, there exists a minimal time $T_{0}$ and a positive constant. $C$ such that

(1.10) $\left(T-T_{0}\right) E_{0} \leq C\left\{\int_{\Sigma_{1}^{+}\left(x_{0}\right)}\left(\gamma \frac{\partial \varphi}{\partial \nu}\right)^{2} d \sigma d t+\int_{\Sigma_{2}^{+}\left(x_{0}\right)}\left(\gamma^{\#} \frac{\partial\left(\gamma_{+} \varphi\right)}{\partial \nu^{\#}}\right)^{2} d \sigma^{\#} d t\right\}$,

where $E_{0}$ denotes the energy of $\varphi$,

$$
\begin{gathered}
\Sigma_{1}^{+}\left(x_{0}\right)=\left\{x \in \Gamma_{1}: m(x) \cdot \nu(x)>0\right\} \times(0, T), \\
\Sigma_{2}^{+}\left(x_{0}\right)=\left\{x^{\#} \in \partial \Gamma: m\left(x^{\sharp}\right) \cdot \nu^{\sharp}\left(x^{\sharp}\right)>0\right\} \times(0, T) .
\end{gathered}
$$

Owing to HUM, we arrive to the exact controllability of (1.8) with Dirichlet data only on $\Sigma_{1}^{+}\left(x_{0}\right)$ and $\Sigma_{2}^{+}\left(x_{0}\right)$.

For convenience, in all this paper, we suppose that $C$ is a positive constant, which may depend on the domain $\Omega$ and on the circumstances but which never depends on the solution of the problem we solve.

\section{VARIATIONAL FORMULATION OF THE PROBLEM}

Let us introduce the two following Hilbert spaces

$$
\begin{gathered}
H_{1}=L^{2}(\Omega) \times L^{2}(\omega), \\
V_{1}=\left\{\vec{u}=\left(u, u_{1}\right) \in H^{1}(\Omega) \times H_{0}^{1}(\omega) \quad\right. \text { satisfying }
\end{gathered}
$$

$$
\begin{gathered}
u=0 \text { on } \Gamma_{1}, \\
\left.\gamma_{+} u=\gamma_{-} u=u_{1} \text { on } \Gamma\right\},
\end{gathered}
$$

this last one being equipped with the norm of $H^{\prime}(\Omega) \times H^{\prime}(\omega)$. Let us recall that $H_{0}^{1}(\omega)$ denotes the closure in $H^{1}(\omega)$ of $\mathscr{Z}(\omega)$ (the space of $C^{\infty}$-functions with compact support in $\omega$ ).

We introduce the form of the gradient on $V_{1}$ as follows : for all $\vec{u}=\left(u, u_{1}\right)$, $\vec{v}=\left(v, v_{1}\right) \in V_{1}$, we set

$$
b(\vec{u}, \vec{v})=\int_{\Omega} \nabla_{n} u \cdot \nabla_{n} v d x+\int_{\omega} \nabla_{n-1} \dot{u}_{1} \cdot \nabla_{n-1} v_{1} d x^{\#}
$$


The form $b$ is obviously a continuous bilinear form on $V_{1} \times V_{1}$; moreover, owing to Poincaré's inequality in $\Omega$ and $\omega$, it is $V_{1}$-coercive i.e. there exists a positive constant $\beta$ such that

$$
b(\vec{u}, \vec{u}) \geq \beta\|\vec{u}\| \stackrel{2}{V_{1}}, \forall \vec{u} \in V_{1} .
$$

Owing to the Lax-Milgram lemma, we obtain the

Lemma 2.1. For all $(f, g) \in H_{1}$, there exists a unique solution $\vec{u} \in V_{1}$ of

$$
b(\vec{u}, \vec{v})=\int_{\Omega} f v d x+\int_{\omega} g v_{1} d x^{\#}, \quad \forall \vec{v}=\left(v, v_{1}\right) \in V_{1} .
$$

Formal applications of Green's formula drive us to say that $\vec{u}=\left(u, u_{1}\right)$ is a weak solution of problem (1.1)-(1.3). Before giving a meaning to (1.1)-(1.3), we first show that problem (2.3) splits into two problems corresponding to (I.4) and (1.5). In dimension 2, this splitting is based on the trace theorem 1.5.2.3 of [5]. In dimension 3 , we use the following one:

Theorem 2.2. Let $\theta$ be a bounded open set of $\mathbf{R}^{3}$, with a polyhedral boundary $\partial \theta, \theta$ lying on only one side of its boundary, and set $\partial \theta=\bigcup_{j=1}^{N} \vec{\Gamma}_{j}$, where $\Gamma_{j}$ are disjoint plane open sets. For all $k=1, \ldots, N$, let us denote by $\gamma_{k}$, the trace operator on the face $\Gamma_{k}$. For a fixed $j \in\{1, \ldots, N\}$, let us set

$$
V_{j}=\left\{u \in H^{l}(\theta): \gamma_{k} u=0, \quad \forall k \neq j\right\} .
$$

Then the operator

$$
u \rightarrow \gamma_{j} u
$$

is a continuous operator from $V_{j}$ into $\tilde{H}^{1 / 2}\left(\Gamma_{j}\right)$. Identifying $\Gamma_{j}$ with a polygonal open set of $\mathbf{R}^{2}$, we recall that (see Definition 1.3.2.5 of [5]) $v \in$ $\tilde{H}^{1 / 2}\left(\Gamma_{j}\right)$ iff $\tilde{v} \in H^{1 / 2}\left(\mathbf{R}^{2}\right)$, where $\tilde{v}$ is the continuation of $v$ by zero outside $\Gamma_{j}$.

Proof: Owing to the results of [4], we know that this operator is continuous and surjective onto the subspace of $f \in H^{1 / 2}\left(\Gamma_{j}\right)$ satisfying

$$
\int_{\Gamma_{j} \times \Gamma_{1}}|f(x)|^{2} \frac{d x d y}{\|\overline{x-y}\|^{3}}<+\infty
$$

for all $l \neq j,\|\cdot\|$ denoting the Euclidean norm of $\mathbf{R}^{3}$. 
We now prove that this condition (2.4) implies that

$$
\int_{\Gamma_{j}}|f(x)|^{2} \frac{d x}{\rho_{j}(x)}<+\infty
$$

where $\rho_{j}(x)=d\left(x, \partial \Gamma_{j}\right)$ is the distance from $x$ to the boundary of $\Gamma_{j}$. This will prove the result, owing to Lemma 1.3.2.6 of [5].

As in [4], by localization and linear change of variables, we are reduced to the case $\theta=G$, where $G$ is the trihedral $x_{1}>0, x_{2}>0, x_{3}>0$. Let us denote by $S_{k}$, the face defined by $x_{k}=0$, for $k \in\{1,2,3\}$. We may suppose that $\Gamma_{j}$ corresponds to $S_{1}$.

By a direct computation, we check that for $x \in S_{1}$, we have

$$
\int_{S_{1}} \frac{d y}{\|x-y\|^{3}} \geq \frac{\pi}{2 x_{l}}
$$

for $l \in\{2,3\}$. Therefore, the conditions (2.4) tanspose to $G$ and the previous inequality imply that

$$
\int_{S_{1}}|f(x)|^{2} \frac{d x}{\rho_{1}(x)}<+\infty
$$

since on $S_{1}, \rho_{1}(x)=\min \left(x_{2}, x_{3}\right)$. This proves the theorem.

Lemma 2.3. Let $\theta=\left\{x=\left(x^{\#}, x_{n}\right) \in \mathbf{R}^{n}: 0<x_{n}<1, x^{\#} \in \theta_{1}\right\}$, where $\theta_{1}$ is an open set of $\mathbf{R}^{n-1}$ with a smooth boundary. Let $\gamma_{n}$ denote the trace operator on the part of the boundary of $\theta$ corresponding to $x_{n}=0$. Let $u \in H^{\prime}(\theta)$. Then

$$
\begin{aligned}
& \gamma_{n} u=0 \text { if and only if } \\
& \int_{\theta}\left|u\left(x^{\#}, x_{n}\right)\right|^{2} \frac{d x^{\#} d x_{n}}{x_{n}}<+\infty
\end{aligned}
$$

Proof: Let $v \in \mathscr{D}(\bar{\theta})$ (the set of $C^{\infty}$-functions in $\bar{\theta}$ ). We can write

$$
v\left(x^{\#}, x_{n}\right)-v\left(x^{\#}, 0\right)=\int_{0}^{x_{n}} \frac{\partial v}{\partial y_{n}}\left(x^{\#}, y_{n}\right) d y_{n} .
$$

Therefore, Schwarz's inequality implies

$$
\left|v\left(x^{\sharp}, x_{n}\right)-\left(\gamma_{n} v\right)\left(x^{\#}\right)\right|^{2} \leq x_{n} \int_{0}^{x_{n}}\left|\frac{\partial v}{\partial y_{n}}\left(x^{\sharp}, y_{n}\right)\right|^{2} d y_{n} .
$$


Dividing this inequality by $x_{n}$ and integrating over $\theta$, we get

$$
\int_{\theta} \frac{1}{x_{n}}\left|v\left(x^{\#}, x_{n}\right)-\left(\gamma_{n} v\right)\left(x^{\sharp}\right)\right|^{2} d x^{\#} d x_{n} \leq\|v\|_{H^{\prime}}^{2}(\theta) .
$$

Since $\mathscr{P}(\bar{\theta})$ is dense in $H^{\mathrm{j}}(\theta)$ (see Theorem 1.4.2.1 of [5]), (2.8) shows that for all $u \in H^{\prime}(\theta)$, the function

$$
\left(x^{\#}, x_{n}\right) \rightarrow \frac{1}{\sqrt{x_{n}}}\left(u\left(x^{\#}, x_{n}\right)-\left(\gamma_{n} u\right)\left(x^{\#}\right)\right)
$$

belongs to $L^{2}(\theta)$ and (2.8) holds for $u$.

Let us fix $u \in H^{\prime}(\theta)$. If $\gamma_{n} u=0$, then (2.8) directly implies (2.7). Reciprocally, if (2.7) holds, then (2.8) and the triangular inequality imply

$$
\int_{\theta} \frac{1}{x_{n}}\left|\left(\gamma_{n} u\right)\left(x^{\#}\right)\right|^{2} d x^{\#} d x_{n}<+\infty
$$

This proves (2.6) since $\frac{1}{x_{n}}$ is not integrable on $] 0,1[$.

Using local charts and Lemma 1.3.2.6 of [5], we obtain the

Corollary 2.4. Let $\theta$ be a bounded open set of $\mathbf{R}^{n}$ with a Lipschitz boundary. Then

$$
\tilde{H}^{1 / 2}(\theta) \cap H^{1}(\theta)=H_{0}^{\mathrm{l}}(\theta)
$$

Lemma 2.5. Let $\omega_{1}=\omega \backslash \bar{\Gamma}$. If $\vec{u}=\left(u, u_{1}\right) \in V_{1}$, then

$$
u_{1} \in H_{0}^{1}(\Gamma) \cap H_{0}^{1}\left(\omega_{1}\right) \text {. }
$$

Proof: Applying Theorem 1.5.2.3 of [5] in dimension 2 and Theorem 2.2 in dimension 3 in the open set $\Omega^{+}=\left\{x \in \Omega: x_{2}>0\right\}$, we deduce that $u_{1}=\gamma_{+} u$ fulfils

$$
\int_{\Gamma}\left|u_{\mathrm{t}}(x)\right|^{2} \frac{d x}{1=x_{1}}<+\infty
$$

since $1-x_{1} \geq d(x, \partial \widetilde{\Gamma})$, where $\tilde{\Gamma}$ is the part of the boundary of $\Omega^{+}$ corresponding to $x_{2}=0$ (notice that $\Gamma \subset \widetilde{\Gamma}$ ). 
If we denote $\widehat{\partial \Gamma}=\left\{x \in \partial \Gamma: x_{1}=1\right\}$ and $\hat{\gamma}$, the trace operator on $\widehat{\partial \Gamma}$ in $\Gamma$, then Lemma 2.3 and inequality (2.10) imply that

$$
\hat{\gamma} u_{1}=0 \text { on } \widehat{\partial \mathrm{T}^{\top}} \text {. }
$$

Since we already know that the trace of $u_{1}$ is equal to zero on $\partial \omega$, we deduce (2.9).

We are now able to state the splitting of problem (2.3). Let us introduce the Hilbert spaces:

$$
\begin{aligned}
& H=L^{2}(\Omega) \times L^{2}(\Gamma) \\
& V=\left\{u \in H^{1}(\Omega) \quad \text { satisfying (2.1) and } \gamma_{+} u=\gamma_{-} u \in H_{0}^{\prime}(\Gamma)\right\},
\end{aligned}
$$

equipped with the norm

$$
\|u\|_{V}=\left(\|u\|_{H^{\prime}(\Omega)}^{2}+\left\|\gamma_{+} u\right\|_{H^{\prime}(\Gamma)}^{2}\right)^{1 / 2} .
$$

We consider the form of the gradient on $V$ as follows : for all $u, v \in V$, we set

$$
a(u, v)=\int_{\Omega} \nabla_{n} u \nabla_{n} v d x+\int_{\Gamma} \nabla_{n-1}\left(\gamma_{+} u\right) \nabla_{n-1}\left(\gamma_{+} v\right) d x^{\#}
$$

As previously, this form is a continuous coercive bilinear form on $V$.

Theorem 2.6. Let $(f, g) \in H_{1}$. Then $\vec{u}=\left(u, u_{1}\right) \in V_{1}$ is the unique solution of (2.3) if and only if

i) $u \in V$ and is the unique solution of

$$
a(u, v)=\int_{\Omega} f v d x+\int_{\Gamma} g \gamma_{+} v d x^{\#}, \quad \forall v \in V .
$$

ii) the restriction $u_{2}$ of $u_{1}$ to $\omega_{1}$ belongs to $H_{0}^{1}\left(\omega_{1}\right)$ and fulfils

$$
\int_{\omega_{1}} \nabla_{n-1} u_{2} \nabla_{n-1} w d x^{\#}=\int_{\omega_{1}} g w d x^{\#}, \forall w \in H_{0}^{1}\left(\omega_{1}\right) .
$$

Remarks 2.7: We see that $u_{2}$ is a solution of a classical Dirichlet problem in a convex domain of $\mathbf{R}^{n-1}$. Owing to the results of [5], $u_{2} \in H^{2}\left(\omega_{1}\right)$ and the exact controllability of the associated wave equation is wellknown (cf. [12]). Therefore, from now on, we only study the solution $u \in V$ of problem (2.12). 
The splitting phenomenon is a consequence of the Dirichlet boundary conditions imposed on $\Gamma_{1}$. For instance if we consider Neumann boundary conditions on $\Gamma_{1}$, no splitting occurs.

In order to show in what sense $u \in V$, solution of (2.12), is a solution of (1.4), we need the extension of Theorems 1.5.3.10 and 1.5.3.11 of [5] in dimension 3 . The proofs are actually identical using the Green formula (22.11) of [2] and Theorem 2.2.

Theorem 2.8. Assume that the assumptions of Theorem 2.2 are fulfilled. For $p>1$, let us set

$$
E\left(\Delta, L^{p}(\theta)\right)=\left\{u \in H^{1}(\theta): \Delta u \in L^{p}(\theta)\right\}
$$

Then the mapping

$$
u-\gamma_{j} \frac{\partial u}{\partial \nu_{j}}
$$

which is defined on $\mathscr{Q}(\bar{\theta})$, has a unique continuous extension as an operator from $E\left(\Delta, L^{p}(\theta)\right)$ into $\left(\tilde{H}^{1 / 2}\left(\Gamma_{j}\right)\right)^{\prime}$. Moreover, the following half Green identity still holds:

$$
\int_{\theta} \Delta u v d x=-\int_{\theta} \nabla u \nabla v d x+\sum_{j=1}^{n}<\gamma_{j} \frac{\partial u}{\partial \nu_{j}} ; \gamma_{j} v>
$$

for all $u \in E\left(\Delta, L^{p}(\theta)\right)$ and all $v \in \mathscr{D}(\bar{\theta})$ such that $\gamma_{j} v \in \mathscr{D}\left(\Gamma_{j}\right)$, for every $j \in\{1, \ldots, N\}$.

Theorem 2.9. Let $(f, g) \in H$ be fixed. Then the unique solution $u \in V$ of (2.12) satisfies

$$
\begin{gathered}
-\Delta_{n} u=f \text { in } \Omega \\
-\Delta_{n-1}\left(\gamma_{+} u\right)+\gamma_{+} \frac{\partial u}{\partial \nu^{+}}+\gamma_{-} \frac{\partial u}{\partial \nu^{-}}=g \text { in } \Gamma .
\end{gathered}
$$

Proof: (2.15) is a direct application of (2.12) with $v \in \mathscr{Q}(\Omega)$. So we deduce that $u \in E\left(\Delta, L^{p}(\Omega)\right)$. But unfortunately, $\Omega$ does not fulfil the assumption of Theorem 2.8. So we use the following trick. Let us set

$$
\begin{gathered}
\hat{\Omega}=\{x \in]-1,1\left[n: x_{1}>0\right\}, \\
\hat{\Omega}^{+}=\left\{x \in \hat{\Omega}: x_{2}>0\right\}, \\
\hat{\Omega}^{-}=\left\{x \in \hat{\Omega}: x_{2}<0\right\} .
\end{gathered}
$$


Then we know that

$$
u \in E\left(\Delta, L^{p}\left(\hat{\Omega}^{+}\right)\right), u \in E\left(\Delta, L^{p}\left(\hat{\Omega}^{-}\right)\right) .
$$

This allows us to give a meaning to $\gamma_{+} \frac{\partial u}{\partial \nu^{+}}$and $\gamma_{-} \frac{\partial u}{\partial \nu^{-}}$as elements of $\left(\tilde{H}^{1 / 2}(\Gamma)\right)^{\prime}$ :

Taking $\varphi \in \mathscr{Z}(\hat{\Omega})$, then $\varphi^{+}$(resp. $\varphi^{-}$) the restriction of $\varphi$ to $\widehat{\Omega}^{+}$(resp. $\hat{\Omega}^{-}$) fulfils the assumptions of Theorem 2.8 in $\hat{\Omega}^{+}$(resp. $\hat{\Omega}^{-}$). Therefore, using the Green identity (2.14) to the pairs $\left(u, \varphi^{+}\right)$in $\hat{\Omega}^{+}$and $\left(u, \varphi^{-}\right)$in $\widehat{\Omega}^{-}$and adding the results, we obtain

$$
\int_{\Omega} \Delta_{n} u \varphi d x=-\int_{\Omega} \nabla_{n} u \nabla_{n} \varphi d x+<\gamma_{+} \frac{\partial u}{\partial \nu^{+}}+\gamma_{-} \frac{\partial u}{\partial \nu^{-}} ; \gamma_{+} \varphi>
$$

Owing to (2.12), (2.15) and the previous identity, we obtain

$$
\begin{gathered}
\int_{\Gamma} \nabla_{n-1}\left(\gamma_{+} u\right) \nabla_{n-1}\left(\gamma_{+} \varphi\right) d x^{\#}+\left\langle\gamma_{+} \frac{\partial u}{\partial \nu^{+}}+\gamma_{-} \frac{\partial u}{\partial \nu} ; \gamma_{+} \varphi>\right. \\
=\int_{\Gamma} g\left(\gamma_{+} \varphi\right) d x^{\#}, \quad \forall \varphi \in \mathscr{Q}(\hat{\Omega}) .
\end{gathered}
$$

This proves (2.16).

\section{REGULARITY OF THE WEAK SOLUTION}

The aim of this paragraph is to prove the expansions (1.6) and (1.7). Let us set

$$
\begin{aligned}
& S_{1}(r, \theta)=\eta(r) r^{1 / 2} \sin \left(\frac{\theta}{2}\right), \\
& S_{2}(r)=-\frac{4}{3} \eta(r) r^{\frac{3}{2}} .
\end{aligned}
$$

Theorem 3.1. Let $u \in V$ be the solution of (2.12) with data $(f, g) \in H$. Then $u$ and $\gamma_{+} u$ admit the following expansion:

$$
u=u_{0}+c S_{1} \text {, }
$$

$$
\gamma_{+} u=u_{20}+c S_{2},
$$


where $u_{0} \in H^{2}(\Omega), u_{20} \in H^{2}(\Gamma)$ and in dimension $2, c \in \mathbf{R}$, while in dimension 3, $c \in H^{1}(\Gamma)$. Moreover, we have the following estimate:

$$
\left\|u_{0}\right\|_{H^{2}(\Omega)}+\left\|u_{20}\right\|_{H^{2}(\Gamma)}+\|c\|_{H^{\prime}(\Gamma)} \leq C\|(f, g)\|_{H} .
$$

In dimension 2, in order to apply the results of Corollary 4.4.1.4 of [5] to $u$, we need to show that $\gamma_{+} u$ belongs to $H^{3 / 2}(\Gamma)$. We use the

Theorem 3.2. Let $\theta$ be a bounded open set of $\mathbf{R}^{m}, m \geq 1$, with a smooth boundary ; $\theta$ being only on one side of its boundary. If $v \in H_{0}^{\prime}(\theta)$ is such that

$$
\Delta_{m} v \in\left(\tilde{H}^{1 / 2}(\theta)\right)^{\prime}
$$

then

$$
v \in H^{3 / 2}(\theta) .
$$

Proof: The proof is made by interpolation and using Theorems 1.6.2 and 1.11 .7 of $[13]$.

Proof of Theorem 3.1 when $n=2:$ Owing to (2.16), $\gamma_{+} u \in H_{0}^{1}(\Gamma)$ fulfils

$$
-\Delta_{n-1}\left(\gamma_{+} u\right)=g-\gamma_{+} \frac{\partial u}{\partial \nu^{\dagger}}-\gamma_{-} \frac{\partial u}{\partial \nu}
$$

which belongs to $\left(\tilde{H}^{1 / 2}(\Gamma)\right)^{r}$. So Theorem 3.2 shows that

$$
\gamma_{+} u \in H^{3 / 2}(\Gamma)
$$

We now remark that $u$ is a solution of the following non-homogeneous Dirichlet problem in $\Omega$ :

$$
\left\{\begin{aligned}
\Delta_{2} u & =-f \in L^{2}(\Omega) \text { in } \Omega \\
u & =0 \text { on } \Gamma_{1}, \\
\gamma_{+} u & =\gamma_{-} u \in H^{3 / 2}(\Gamma) \cap H_{0}^{j}(\Gamma) \text { on } \Gamma
\end{aligned}\right.
$$

Owing to Corollary 4.4.4.14 and Remark 4.4.4.16 of [5] (see also Theorem 1.4.10 of [2]), we obtain the decomposition (3.3) for $u$. 
Using this expansion (3.3) of $u$ in the right-hand side of (3.6) and computing the normal derivative of the singular part, we see that $\gamma_{+} u \in H_{0}^{1}(\Gamma)$ fulfils

$$
-\Delta_{1}\left(\gamma_{+} u\right)=g_{1}+c \eta x_{1}^{-1 / 2},
$$

where we set

$$
g_{1}=g-\gamma_{+} \frac{\partial u_{0}}{\partial \nu^{+}}-\gamma_{-} \frac{\partial u_{0}}{\partial \nu^{-}} \in L^{2}(\Gamma)
$$

Remark that $x_{1}^{-1 / 2}$ does not belong to $L^{2}(\Gamma)$. But we can compute explicitly the solution $w$ of

$$
\left\{\begin{array}{l}
\left.-\frac{\partial^{2} w}{\partial x_{1}^{2}}=x_{1}^{T 2} \text { on }\right] 0, \mathrm{I}[ \\
w(0)=\frac{\partial w}{\partial x_{1}}(0)=0 .
\end{array}\right.
$$

This is $w\left(x_{1}\right)=-\frac{4}{3} x_{1}^{3 / 2}$.

Finally, the function

$$
u_{20}=\gamma_{+} u-c \eta w
$$

belongs to $H_{0}^{1}((0,1))$ and fulfils

$$
-\Delta_{1} u_{20} \in L^{2}((0,1)) .
$$

So $u_{20} \in H^{2}((0,1))$ and the expansion (3.4) follows from (3.7).

By Theorem 3.5 hereafter (applied with $\lambda=0$, see Remarks 3.6), there exists $\left(K_{1}, K_{2}\right) \in H$ such that

$$
c=\left((f, g),\left(K_{1}, K_{2}\right)\right)_{H},
$$

where $(\cdot,)_{H}$ denotes the inner product of $H$.

Let us define the linear operator

$$
T: H \rightarrow H^{2}(\Omega) \times H^{2}(\Gamma):(f, g) \rightarrow\left(u_{0}, u_{20}\right) .
$$


Owing to the closed graph theorem, if $T$ is closed, then $T$ is continuous and therefore the estimate (3.5) holds. But the closeness of $T$ is a direct consequence of (3.8) and the continuous dependence of the solution $u \in V$ of (2.12) with respect to $(f . g)$ in $H$.

This proves Theorem 3.1 when $n=2$.

In order to obtain an analogous decomposition result in dimension 3, we need to study "the Helmoltz equation" in dimension 2. Actually, we shall extend the results of $\S 2$ of $[6]$ in our situation.

Theorem 3.3. Assume that $n=2$ and let $(f, g) \in H$ and $\lambda$ be a real parameter $\geq 1$. We denote by $u$, the unique solution of

$$
a(u, v)+\lambda\left(\left(u, \gamma_{+} u\right),\left(v, \gamma_{+} v\right)\right)_{H}=\left((f, g),\left(v, \gamma_{+} v\right)\right)_{H}, \quad \forall v \in V .
$$

Then $u$ and $\gamma_{+} u$ admit the following expansion

$$
\begin{gathered}
u=u_{R}+c e^{-r \sqrt{\lambda}} S_{1}, \\
\gamma_{+} u=u_{2 R}+c e^{-r \sqrt{\lambda}} S_{2},
\end{gathered}
$$

where $u_{R} \in H^{2}(\Omega), u_{2} \in H^{2}(\Gamma)$, and $c \in \mathbf{R}$ satisfy the estimates

$$
\begin{gathered}
\sum_{i=0}^{2} \lambda^{\frac{2-i}{2}}\left\{\left\|u_{R}\right\|_{t f^{i}(\Omega)}+\left\|u_{2 R}\right\|_{H^{i}(\Gamma)}\right\} \leq C\|(f, g)\|_{H}, \\
|c| \leq C \lambda^{-\frac{1}{4}\|(f, g)\|_{H},}
\end{gathered}
$$

where $C$ does not depend on $\lambda$.

Proof: The idea of the proof is identical with Theorem 2.1 of [6] but the technics are different. The main step is based on an explicit formula for $c$, obtained as in [3].

Applying (3.9) with $v=u$, we can show that $u$ fulfils

$$
\sqrt{\lambda}\|u\|_{V}+\lambda\left\|\left(u, \gamma_{+} u\right)\right\|_{H} \leq C\|(f, g)\|_{j} .
$$

Since $u \in V$ may be seen as a solution of (2.12) with a datum $(f-\lambda u$, $\left.g-\lambda \gamma_{+} u\right)$ in $H$, Theorem 3.1 shows that $u$ and $\gamma_{+} u$ admit the respective expansion (3.3) and (3.4); moreover the estimate (3.5) becomes

$$
\left\|u_{0}\right\|_{H^{2}(\Omega)}+\left\|u_{20}\right\|_{H^{2}(\Gamma)}+|c| \leq C\left\{\|(f, g)\|_{H}+\lambda\|(u, \gamma+u)\|_{H}\right\} .
$$


Owing to (3.14), we deduce that

$$
\left\|u_{0}\right\|_{H^{2}(\Omega)}+\left\|u_{20}\right\|_{H^{2}(\Gamma)}+|c| \leq C\|(f \cdot g)\|_{H} .
$$

Unfortunately, this estimate does not allow us to prove the estimates (3.12) and (3.13). So as in [6], we modify the decompositions (3.3) and (3.4) of $u$ and $\gamma_{+} u$. Setting

$$
\begin{gathered}
u_{R}=u_{0}+c\left(1-e^{-r \sqrt{\lambda}}\right) S_{1}, \\
u_{2 R}=u_{20}+c\left(1-e^{-r \sqrt{\lambda}}\right) S_{2},
\end{gathered}
$$

we directly obtain (3.10) and (3.11) (with the same constant $c$ as in (3..4)). Moreover, the regularity of $u_{R}$ and $u_{2 R}$ follows from the following lemma, which is proven by an easy computation.

Lemma 3.4. Let $n=2$. Then the functions $\left(1-e^{-r \sqrt{\lambda}}\right) S_{1},\left(1-e^{-r \sqrt{\lambda}}\right) S_{2}$ belong respectively to $H^{2}(\Omega)$ and $H^{2}(\Gamma)$ and fulfil

$$
\begin{gathered}
\left\|\left(1-e^{-r \sqrt{\lambda}}\right) S_{1}\right\|_{H^{2}(\Omega)} \leq C \lambda^{1 / 4}, \\
\left\|\left(1-e^{-r \sqrt{\lambda}}\right) S_{2}\right\|_{H^{2}(\Gamma)} \leq C .
\end{gathered}
$$

It remains to prove (3.12) and (3.13). To do that, we need the explicit formula for $c$. Let us set

$$
\psi(r, \theta)=e^{-r \sqrt{\lambda}} r^{\frac{-1}{2}} \sin \left(\frac{\theta}{2}\right) .
$$

We check that

$$
\begin{gathered}
\left(-\Delta_{2}+\lambda\right) \psi=0 \\
\left\|\left(-\Delta_{2}+\lambda\right)(\eta \psi)\right\|_{L^{2}(\Omega)} \leq C e^{-d \sqrt{\lambda}}
\end{gathered}
$$

for some $d>0$. But the main problem is that

$$
\gamma_{+} \frac{\partial \psi}{\partial \nu^{+}}+\gamma_{-} \frac{\partial \psi}{\partial \nu^{-}}=-e^{-r \sqrt{\lambda}} r^{-3 / 2}
$$

does not belong to $H^{-1}(\Gamma)$. Fortunately, the function $\phi$, defined by

$$
\phi(r, \theta)=4 \eta(r) r^{\frac{1}{2}}\left|\cos \left(\frac{\theta}{2}\right)\right| e^{-r \sqrt{\lambda}},
$$


belongs to $H^{\prime}(\Omega)$ (owing to Theorems 1.4.5.3 and 1.7.1 of [5]) and fulfils

$$
\gamma_{+} \phi=\gamma_{-} \phi=4 \eta r^{\frac{1}{2}} e^{-r \sqrt{\lambda}}
$$

Therefore, on $\Gamma$, the laplacian of $\gamma_{+} \phi$ is equal to $-\eta r^{-3 / 2} e^{-r \sqrt{\lambda}}$, modulo $H^{-1}(\Gamma)$.

Let $\phi_{1} \in V$ be the unique solution of

(3.20) $a\left(\phi_{1}, \nu\right)+\lambda\left(\left(\phi_{1}, \gamma_{+} \phi_{1}\right),\left(\nu, \gamma_{+} \nu\right)\right)_{H}$

$$
\begin{aligned}
& =\int_{\Omega}\left\{\left(\Delta_{2}-\lambda\right)(\eta \psi) v-\nabla_{2} \phi \nabla_{2} v-\lambda \phi v\right\} d x \\
& +\int_{\Gamma}\left\{4 x_{1}^{1 / 2}\left(\Delta_{1}-\lambda\right)\left(\eta e^{-x_{1} \sqrt{\lambda}}\right)+4 x_{1}^{-1 / 2} \frac{\partial\left(\eta e^{-x_{4}} \sqrt{\lambda}\right)}{\partial x_{1}}\right\} \gamma_{+} v d x_{1},
\end{aligned}
$$

for all $v \in V$.

Let us notice that the right-hand side of (3.20) is well defined since $\left(\Delta_{2}-\lambda\right)$ $(\eta \psi) \in L^{2}(\Omega), \phi \in H^{1}(\Omega)$ and because $\gamma_{+} v \in H_{0}^{1}(\Gamma)$ fulfils $x_{1}^{-1} \gamma_{+} v \in L^{2}(\Gamma)$.

Now, we are able to give the expression for $c$.

Theorem 3.5. Under the assumptions of Theorem 3.3, if we set

$$
K=\eta \psi+\phi+\phi_{1},
$$

then we have

$$
-\pi c=\int_{\Omega} f K d x+\int_{\Gamma} g \gamma_{+} K d x_{1} .
$$

Before giving the proof of $(3.22,1.1, \ldots$ how how it implies (3.12) and

To obtain the estimate (3.13), it suffices to estimate the norm of $K$ in $L^{2}(\Omega)$ and the norm of $\gamma_{+} K$ in $L^{2}(\Gamma)$. But we easily check that

$$
\begin{aligned}
& \|\eta \psi\|_{L^{2}(\Omega)} \leq C \lambda^{-1 / 4} \\
& \|\phi\|_{L^{2}(\Omega)} \leq C \lambda^{-3 / 4}, \\
& \left\|\gamma_{+} \phi\right\|_{L^{2}(\Gamma)} \leq C \lambda^{-1 / 2} .
\end{aligned}
$$


Moreover, since $\phi_{1}$ is a solution of $(3.20)$, we can prove that

$$
\left\|\phi_{1}\right\|_{\nu}+\sqrt{\lambda}\left\|\left(\phi_{1}, \gamma_{+} \phi_{1}\right)\right\|_{H} \leq C .
$$

Using the expansion (3.2I) of $K$ and the four previous inequalities, we get

$$
\left\|\left(K, \gamma_{+} K\right)\right\|_{H} \leq C \lambda^{-1 / 4} \text {. }
$$

So (3.22), (3.23) and Schwarz's inequality imply (3.13).

Let us now prove (3.12). Using (3.16), (3.17), Lemma 3.4 and the estimates (3.13) and (3.15), we obtain:

$$
\left\|u_{R}\right\|_{H^{2}(\Omega)}+\left\|u_{2 R}\right\|_{H^{2}\left(I^{\prime}\right)} \leq C\|(f, g)\|_{H} .
$$

Owing to (3.10) and (3.11), we can say that

$$
\begin{aligned}
& \sum_{i=0}^{1} \lambda_{2}^{2-i}\left\{\left\|u_{R}\right\|_{n^{(i(\Omega)}}+\left\|u_{2 R}\right\|_{\mu^{(i)}(\Gamma)}\right\} \\
& \leq \sum_{i=0}^{i} \lambda^{2-i}\left[\|u\|_{H^{i}(\Omega)}+\left\|\gamma_{+} u\right\|_{\mu^{\prime}\left({ }^{\prime}\right)}\right. \\
& \left.+|c|\left\{\left\|e^{-r \sqrt{\lambda}} S_{1}\right\|_{H^{i}(\Omega)}+\left\|e^{-r \sqrt{\lambda}} S_{2}\right\|_{H^{\prime}\left(\Gamma^{\prime}\right)}\right\}\right] \text {. }
\end{aligned}
$$

But we easily check that for $i \in\{0,1\}$ :

$$
\begin{gathered}
\left\|e^{-r \sqrt{\lambda}} S_{1}\right\|_{H^{i}(\Omega)} \leq C \lambda^{-\frac{3}{4}+\frac{j}{2}}, \\
\left\|e^{-r \sqrt{\lambda}} S_{2}\right\|_{H^{i}(\Gamma)} \leq C \lambda \frac{i-2}{2} .
\end{gathered}
$$

Using (3.14), (3.13), (3.26) and (3.27);(3.25) becomes

$$
\sum_{i=0}^{1} \lambda^{\frac{2-i}{2}}\left\{\left\|u_{R}\right\|_{H^{i}(\Omega)}+\left\|u_{2 R}\right\|_{H^{i}(\Gamma)}\right\} \leq C\|(f, g)\|_{H} .
$$

The sum of (3.24) and (3.28) yields (3.12).

Proof of Theorem 3.5: Let us denote by $I$, the right-hand side of (3.22). Using the expansion (3.21) of $K, I$ splits as follows:

$$
I=I_{0}+I_{1}+I_{2}
$$


where we set

$$
\begin{aligned}
& l_{0}=\int_{\Omega} f \eta \psi d x \\
& l_{1}=\int_{\Omega} f \phi d x+\int_{\Gamma} g \gamma_{+} \phi d x_{1}, \\
& I_{2}=\int_{\Omega} f \phi_{1} d x+\int_{\Gamma} g \gamma_{+} \phi_{1} d x_{1} .
\end{aligned}
$$

i) Transformation of $I_{2}: I_{2}$ is the right-hand side of (3.9) with $v=\phi_{1}$, since $\phi_{1} \in V$, we get

$$
I_{2}=a\left(\phi_{1}, u\right)+\lambda\left(\left(\phi_{1}, \gamma_{+} \phi_{1}\right),\left(u, \gamma_{+} u\right)\right)_{H} .
$$

ii) Transformation of $I_{0}$ :The splitting (3.3) of $u$ induces the following one for $I_{0}$ :

$$
I_{0}=T_{0}+c S_{0}
$$

with

$$
\begin{aligned}
& T_{0}=\int_{\Omega}\left(-\Delta_{2}+\lambda\right) u_{0} \eta \psi d x, \\
& S_{0}=\int_{\Omega}\left(-\Delta_{2}+\lambda\right) S_{1} \eta \psi d x .
\end{aligned}
$$

Now, our goal is to use Green's formula in the right-hand side of (3.32) and (3.33).

a) Since $u_{0} \in H^{2}(\Omega)$ and fulfils $u_{0}(0)=0$, Theorem 4.3.2.2 of [5] implies that $u_{0} \in P^{2, p}(\Omega)$, for all $p<2$ (see Definition 4.3.2.l of [5]). Moreover, we check that $\eta \psi \in L^{q}(\Omega)$, for all $q<4$. Since $\psi$ is smooth far from 0 , $\Delta(\eta \psi) \in L^{q}(\Omega)$, for all $q<4$. Therefore, the pair $(\eta \psi, u)$ fulfils the assumptions of Theorem 12.3 of [3] with some $p<2$ and $q \in] 2,4$ [ such that $1 / p+1 / q=1$. Applying the Green formula (12.4) of [3] to this pair, we get

$$
T_{0}=\int_{\Omega} u_{0}\left(-\Delta_{2}+\lambda\right)(\eta \psi) d x+<\gamma_{+} \frac{\partial(\eta \psi)}{\partial \nu^{+}}+\gamma_{-} \frac{\partial(\eta \psi)}{\partial \nu^{-}} ; \gamma_{+} u_{0}>
$$

b) To transform $S_{0}$, we apply Green's formula in $\Omega_{\mathrm{c}}=\Omega \backslash B(0, \varepsilon)$, for all $\varepsilon>0$. By a classical computation, letting $\varepsilon$ go to zero, we obtain

$$
S_{0}=\int_{\Omega} S_{1}\left(-\Delta_{2}+\lambda\right)(\eta \psi) d x-\pi .
$$


In (3.31), replacing $T_{0}$ and $S_{0}$ by their expression (3.34) and (3.35) and using again the expansion (3.3) of $u$, we obtain

$$
I_{0}=\int_{\Omega} u\left(-\Delta_{2}+\lambda\right)(\eta \psi) d x+<\gamma_{+} \frac{\partial(\eta \psi)}{\partial \nu^{+}}+\gamma_{-} \frac{\partial(\eta \psi)}{\partial \nu^{-}} ; \gamma_{+} u>-\pi c
$$

iii) Transformation of $I_{1}$ : The first step consists in using the half Green formula in $\Omega$. Since $u \notin H^{2}(\Omega)$, we again use its expansion (3.3) and we decompose $\int_{\Omega} f \phi d x$ in two integrals corresponding to the regular part and the singular one. For the regular part, we may apply the half Green formula. For the singular part, we use the half Green formula in $\Omega_{\mathrm{c}}$, for $\varepsilon>0$; letting $\varepsilon$ go to zero and using the definition of $\phi$, we obtain

$$
I_{1}=\int_{\Omega}\left(\nabla_{2} u \nabla_{2} \phi+\lambda u \phi\right) d x+\int_{\Gamma}\left(-\Delta_{\mathrm{l}}+\lambda\right)\left(\gamma_{+} u\right) \gamma_{+} \phi d x_{1} .
$$

We now transform the integral over $\Gamma$ by using Green formula in $\Gamma_{\varepsilon}=\Gamma \backslash B(0, \varepsilon)$, for $\varepsilon>0$; and by letting $\varepsilon$ go to zero. Computing the laplacian of $\gamma_{+} \phi$, we get

$$
\begin{aligned}
I_{1}= & \int_{\Omega}\left(\nabla_{2} u \nabla_{2} \phi+\lambda u \phi\right) d x-<\gamma_{+} \frac{\partial(\eta \psi)}{\partial \nu^{+}}+\gamma_{-} \frac{\partial(\eta \psi)}{\partial \nu^{-}} ; \gamma_{+} u> \\
& +\int_{\Gamma}\left\{4 x_{1}^{1 / 2}\left(-\Delta_{1}+\lambda\right)\left(\eta e^{-x_{1} \sqrt{\lambda}}\right)=4 x_{-1 / 2}^{-1 / 2\left(\eta e^{-x_{1} \sqrt{\lambda}}\right)} \frac{\partial x_{1}}{\partial x_{+}} u d x_{1} .\right.
\end{aligned}
$$

iv) Conclusion: In (3.29), replacing $I_{0}, I_{1}$ and $I_{2}$ respectively by (3.36), (3.38) and (3.30) and using the fact that $\phi_{\mathrm{t}}$ is solution of (3.20), we arrive to (3.22)

Remarks 3.6: In view of the proof of (3.22), we need to introduce $\phi$ because, in the right-hand side of (3.36), the duality bracket has a meaning since $\gamma_{+} u=\gamma_{+} u_{0}$ belongs to the weighted space $P^{2-1 / p, p}(\Gamma)$, for some $p<2$; but it has no meaning if we only know that $\gamma_{+} u$ belongs to $H_{0}^{1}(\Gamma)$. Indeed, if the duality bracket would have a meaning for $\gamma_{+} u$ in $H_{0}^{\prime}(\Gamma)$, it would be sufficient to consider $\phi_{1} \in V$, solution of

$$
\begin{aligned}
& a\left(\phi_{1}, v\right)+\lambda\left(\left(\phi_{1}, \gamma_{+} \phi_{1}\right),\left(\nu, \gamma_{+} \nu\right)\right)_{H} \\
= & \int_{\Omega}\left(\Delta_{2}-\lambda\right)(\eta \psi) v d x-<\gamma_{+} \frac{\partial(\eta \psi)}{\partial \nu^{+}} t_{-} \gamma_{-} \frac{\partial(\eta \psi)}{\partial \nu^{-}} ; \gamma_{+} v>\text {, for all } v \in V .
\end{aligned}
$$

Theorem 3.6 is also true if we suppose that $\lambda=0$. Therefore, it gives an explicit formula for the coefficient $c$ of the singularities of the solution $u$ of $(2.12)$ 
Let us suppose that $n=3$ up to the end of paragraph 3. In order to prove Theorem 3.1, we shall develop $u$ in Fourier series with respect to the basis $\left\{\sin \frac{k \pi}{2}\left(x_{3}-1\right)\right\}_{k \in w^{*} .}$ It corresponds to take the partial Fourier transform in the $x_{3}$-variable of the solution of the Dirichlet problem in a polyhedral cylinder $\theta \times \mathbf{R}$, where $\theta$ is a plane polygon of $\mathbf{R}^{2}$, as $\mathbf{P}$. Grisvard did in [6].

We use the following notation:

$$
\left.\Omega=\Omega^{(2)} \times\right]-1,1\left[, \Gamma=\Gamma^{(2)} \times\right]-1,1[,
$$

where $\Omega^{(2)}$ (resp. $\Gamma^{(2)}$ ) is the domain $\Omega$ (resp. $\Gamma$ ) defined in $\S 1$ when $n=2$. Moreover, for a point $x$ in $\Omega$ or in $\Gamma$, we denote

$$
x=\left(x^{(2)}, x_{3}\right) \text {, }
$$

where $x^{(2)}$ belongs to $\Omega^{(2)}$ or $\Gamma^{(2)}$ and $\left.x_{3} \in\right]-1,1[$.

For an arbitrary function $w$ in $L^{2}(\Omega)$ or $L^{2}(\Gamma)$ and for all $k \in \mathrm{N}^{*}$, we set

$$
w_{k}\left(x^{(2)}\right)=\int_{-1}^{+1} w\left(x^{(2)}, x_{3}\right) \sin \frac{k \pi}{2}\left(x_{3}-1\right) d x_{3},
$$

its Fourier coefficient with respect to $\sin \frac{k \pi}{2}\left(x_{3}-1\right)$.

We finally denote by $V^{(2)}$, the Hilbert space $V$ defined on $\Omega^{(2)}$.

We can easily prove the

Lemma 3.7. For all $w \in V$ and all $k \in \mathbf{N}^{*}$, we have

$$
w_{k} \in V^{(2)} \text { and } \gamma_{+} w_{k}=\gamma_{-} w_{k}=\left(\gamma_{+} w\right)_{k} .
$$

We are now able to give the

Proof of Theorem 3.1 when $n=3$ : Let us fix $k \in \mathbf{N}^{*}$. Using Fubini's theorem, we can prove that $u_{k} \in V^{(2)}$ is the unique solution of

$$
\begin{gathered}
\int_{\Omega^{(2)}}\left\{\nabla_{2} u_{k} \nabla_{2} v+\frac{k^{2} \pi^{2}}{-4} u_{k} v\right\} d x^{(2)} \\
+\int_{\Gamma^{(2)}}\left\{\nabla_{1}\left(\gamma_{+} u_{k}\right) \nabla_{1}(\gamma+v)+\frac{k^{2} \pi^{2}}{4} \gamma_{+} u_{k} \gamma+v\right\} d x^{(2) H} \\
=\int_{\Omega^{(2)}} f_{k} v d x^{(2)}+\int_{\Gamma^{(2)}} g_{k} \gamma_{+} v d x^{(2) \#}, \quad \forall v \in V^{(2)} .
\end{gathered}
$$


This shows that $u_{k} \in V^{(2)}$ is the solution of the Helmoltz equation (3.9) with $\lambda=\frac{k^{2} \pi^{2}}{4} \geq \mathrm{I}$ and a datum $\left(f_{k}, g_{k}\right)$. By Theorem 3.3 , there exist $u_{R k} \in H^{2}\left(\Omega^{(2)}\right)$, $u_{2 R k} \in H^{2}\left(\Gamma^{(2)}\right)$ and $c_{k} \in \mathbf{R}$ such that

$$
\begin{gathered}
u_{k}=u_{R k}+c_{k} e^{-r \frac{k \pi}{2}} S_{1}, \\
\gamma_{+} u_{k}=u_{2 R k}+c_{k} e^{-r \frac{k \pi}{2}} S_{2} .
\end{gathered}
$$

Moreover, there exists a constant $C$ independent of $k$ such that

$$
\begin{aligned}
& \left.\sum_{i=0}^{2} k^{2-i}\left\{\left\|u_{R k}\right\|_{H^{i}\left(\Omega^{(2)}\right)}+\left\|u_{2 R k}\right\|_{/ r^{i}\left(\Gamma^{(2)}\right)}\right\}+k^{1 / 2} \mid c_{k} \|\right\} \\
& \leq C\left\{\left\|f_{k}\right\|_{L^{2}\left(\Omega^{(2)}\right)}+\left\|g_{k}\right\|_{L^{2}\left(\Gamma^{(2)}\right)}\right\} .
\end{aligned}
$$

Let us denote by $u_{0}, u_{20}$ and $c$, the functions defined respectively in $\Omega$, $\Gamma$ and also $\Gamma$, with respective Fourier coefficients $u_{R k}, u_{2 R k}$ and $c_{k} e^{-\frac{k \pi}{2}}$. The last function $c$ is actually the convolution $K * \phi$ in $[-1,1]$ (with respect to $x_{3}$ ) of the function $\phi$ and the kernel $K$ with respective coefficients $c_{k}$ and $e^{-r k \pi / 2}$.

The estimate (3.41) precisely shows that $u_{0} \in H^{2}(\Omega), u_{20} \in H^{1}(\Gamma)$ and $c \in H^{1}(\Gamma)$ and fulfil the estimate (3.5). Finally, the expansions (3.39) and (3.40) imply (3.3) and (3.4). This completes the proof of Theorem 3.1.

Remark 3.8: In view of the definition of $c$, it is defined on $\left.\mathbf{R}^{+} \times\right]-1,1[$ and is a solution of

$$
\left\{\begin{array}{l}
\left.\Delta_{2} c=0 \text { in } \mathbf{R}^{+} \times\right]-1,1[, \\
c(0, \cdot)=\phi \text { in }]-1,1[, \\
c(\cdot, 1)=c(\cdot,-1)=0 \text { on } \mathbf{R}^{+} .
\end{array}\right.
$$

This means that $c$ is the solution of the Dirichlet problem in $\left.\mathbf{R}^{+} \times\right]-1,1[$ with a boundary datum $\phi \in H^{1 / 2}(]-1,1[$ ) (owing to the estimate (4.4)). So the regularity of $c$ is in accordance with the regularity we expected. Moreover, far from $x_{1}=0, c$ is smooth.

In $\S 5$, we shall need the following estimate about $c$ and $\nabla_{2} c$ near $x_{1}=0$. 
Lemma 3.9. Assume that $n=3$. Then under the assumptions of Theorem 3.1, for all $\varepsilon \in] 0,1[$, we have:

$$
\begin{aligned}
& \int_{-1}^{+1}\left|c\left(\varepsilon, x_{3}\right)\right|^{2} d x_{3} \leq C\|(f, g)\|_{H}^{2}, \\
& \int_{-1}^{+1}\left|\nabla_{2} c\left(\varepsilon, x_{3}\right)\right|^{2} d x_{3} \leq \frac{C}{\varepsilon}\|(f, g)\|_{H}^{2} .
\end{aligned}
$$

Proof: Since the Fourier coefficients of $c$ are $c_{k} e^{-r \frac{k \pi}{2}}$, by Parseval's identity, we have

$$
\begin{aligned}
& \int_{-1}^{+1}\left|c\left(\varepsilon, x_{3}\right)\right|^{2} d x_{3}=\sum_{k \in \mathrm{N}^{*}}\left|c_{k}\right|^{2} e^{-\varepsilon k \pi} . \\
& \int_{-1}^{+1}\left|\nabla_{2} c\left(\varepsilon, x_{3}\right)\right|^{2} d x_{3} \leq C \sum_{k \in \aleph^{*}}\left|c_{k}\right|^{2} k^{2} e^{-\varepsilon k \pi} .
\end{aligned}
$$

The inequality (3.41) implies that

$$
\sum_{k \in N^{*}}\left|c_{k}\right|^{2} k \leq C\|(f, g)\|_{H}^{2} .
$$

The inequalities (3.42) and (3.43) are a direct consequence of (3.44)-(3.46) since we remark that

$$
e^{-\varepsilon k \pi} \leq C k, k e^{-\varepsilon k \pi} \leq \frac{C}{\varepsilon} .
$$

To end this section, we shall prove that the outward normal derivative $\gamma^{\sharp} \frac{\partial(\gamma+\underline{u})}{\partial \nu^{\#}}$ of $\gamma_{+} u$ on $\partial \Gamma$ exists and belongs to $L^{2}(\Gamma)$.

Theorem 3.10. Under the assumptions of Theorem 3.1, we have

$$
\gamma^{\sharp} \frac{\partial(\gamma+u)}{\partial \nu^{\#}} \in L^{2}(\partial \Gamma)
$$

and fulfils

$$
\left\|\gamma^{\#} \frac{\partial(\gamma+u)}{\partial \nu^{\#}}\right\|_{L^{2}(\partial \Gamma)} \leq C\|(f, g)\|_{H} .
$$


Proof: When $n=2$, since $c$ is a constant, the claim follows from (3.5) and the Sobolev imbedding theorem.

Let us assume that $n=3$. We prove that $\gamma_{+} u \in W^{2 . p}(\Gamma)$, for all $p<2$ and fulfils

$$
\left\|\gamma_{+} u\right\|_{W^{2, p}(\Gamma)} \leq C\|(f, g)\|_{H},
$$

where $C$ may depend on $p$, for all $p<2$.

Using the expansion (3.4) of $\gamma_{+} u$ and the definitions of $S_{2}$ and $c$, we see that

$$
\Delta_{2}\left(\gamma_{+} u\right)=h_{1}-c \eta x_{1}^{-1 / 2},
$$

where $h_{1} \in L^{2}\left(I^{\prime}\right)$ and

$$
\left\|h_{1}\right\|_{L^{2}(\Gamma)} \leq C\|(f, g)\|_{H} .
$$

Since $x_{1}^{-1 / 2} \in L^{p}(\Gamma)$, for all $p<2$, we deduce that $\Delta_{2}\left(\gamma_{+} u\right) \in L^{p}(\Gamma)$, for all $p<2$ and using (3.5), we have

$$
\left\|\Delta_{2}\left(\gamma_{+} u\right)\right\|_{L^{\prime} m} \leq C\|(f, g)\|_{H} .
$$

As $\gamma_{+} u \in H_{0}^{\mathrm{l}}(\Gamma)$ and $\Gamma$ is a convex domain of the plane, we deduce (3.48) (see for instance Theorem 4.4.3.7 of [5]).

Using the trace theorem 1.5.2.1 of [5], the Sobolev imbedding theorem and the estimate (3.48), we arrive to (3.47).

\section{THE WAVEE EQUATION}

Our first aim is to establish the existence of a solution to the wave equation associated with the boundary value problem (1.4). The easiest manner is to introduce a selfadjoint operator $A$ from $H$ into $H$ associated with (1.4).

The bilinear form a induces an isomorphism $Q$ from $V$ into $V^{\prime \prime}$ defined by

$$
(\propto / u)(v)=a(u, v), \quad \forall u, v \in V .
$$

Using the density of $\mathscr{Z}(\Gamma)$ in $L^{2}(\Gamma)$ and the density of $\mathscr{Z}(\Omega)$ in $L^{2}(\Omega)$, we can show that $V$ is dense in $H$. Moreover, the Rellich-Kondrachov theorem implies that $V$ is compactly imbedded into $H$. Therefore, the bilinear form a 
also induces a positive selfadjoint operator $A$ from $H$ into $H$, with a compact inverse, defined by

$$
\left\{\begin{array}{l}
D_{A}=\{u \in V: \propto \mathscr{A} u \in H\} \\
\forall u \in D_{A}: A u=\left(-\Delta_{n} u,-\Delta_{n-1}\left(\gamma_{+} u\right)+\gamma_{+} \frac{\partial u}{\partial \nu^{+}}+\gamma_{-} \frac{\partial u}{\partial \nu^{-}}\right)
\end{array}\right.
$$

owing to Theorem 2.9. It is also wellknown that $D_{A}$ is dense in $V$ and $D_{A^{1 / 2}}=V$ (see [10], for instance).

The wave equation associated with $A$ may be written as follows : given $\varphi_{0} \in V, \varphi_{1} \in H$ and $f \in L^{\prime}(0, T ; H)$, find a solution $\varphi$ of

$$
\left\{\begin{array}{l}
\varphi \in C\left([0, T], V \cap C^{\prime}([0, T], H)\right. \\
\varphi^{\prime \prime}(t)+A \varphi(t)=f(t), t \in[0, T] \\
\varphi(0)=\varphi_{0} \\
\varphi^{\prime}(0)=\varphi_{l}
\end{array}\right.
$$

where $\varphi^{\prime}$ denotes the derivative of $\varphi$ with respect to the variable $t$.

Since $A$ admits a sequence of eigenfunctions, which forms a basis of $H$, Theorem 3.1 of [7] can be adapted to our setting. Therefore, we have the

Theorem 4.1. Let $\varphi_{0} \in D_{A^{\pi}}, \varphi_{1} \in D_{A^{s-1 / 2}}$ and $f \in L^{1}\left(0, T ; D_{A^{s-1 / 2}}\right)$ with $s \geq 1 / 2$. Then problem (4.1) has a unique solution $\varphi \in C\left([0,7], D_{A} s\right) \cap$ $C^{l}\left([0, T], D_{A^{s-1 / 2}}\right)$ fulfilling

(4.2) $\|\varphi\|_{C\left([0, T], D_{\left.A^{3}\right)}\right.}+\|\varphi\|_{C^{\prime}\left([0, T], D_{\left.A^{3}-1,2\right)}\right.}$

$$
\leq C\left\{\left\|\varphi_{0}\right\|_{D_{A^{*}}}+\left\|\varphi_{1}\right\|_{D_{A^{x}-1 / 2}}+\|f\|_{L^{\prime}\left(0, T: D_{A^{3}-1,2,1}\right\}}\right\}
$$

In the same way, Remark 3.2 of [7] still holds.

Theorem 4.2. Let $\varphi_{0} \in V, \varphi_{1} \in H$ and $f \in L^{1}(0, T ; H)$ and let $\varphi$ be the unique solution of (4.1). Then there exist sequences $\varphi_{0 m} \in D_{A}, \varphi_{1 m} \in V, f_{m} \in$ $C([0,7], V), m \in \mathbf{N}^{*}$, such that

$$
\begin{gathered}
\varphi_{0 m} \rightarrow \varphi_{0} \text { in } V, \\
\varphi_{1 m}-\varphi_{\mid} \text {in } H, \\
f_{m \rightarrow f} \text { in } L^{1}(0, T ; H) \text {, as } m \rightarrow+\infty .
\end{gathered}
$$


Moreover, the solution $\varphi_{m}$ of (4.I) with data $\varphi_{0 m}, \varphi_{1 m}, f_{m}$ fulfils

$$
\varphi_{m} \in C\left([0, T], D_{A}\right) \cap C^{1}([0, T], V) \cap C^{2}([0, T], H),
$$

and

$$
\varphi_{m} \rightarrow \varphi \quad \text { in } \quad C([0, T], V) \cap C^{1}([0, T], H) \text {, as } \dot{m} \rightarrow+\infty \text {. }
$$

Proof: Direct consequence of the density of $\mathscr{B}(0, T ; V)$ in $L^{1}(0, T ; H)$.

We now establish a law of conservation of energy.

Theorem 4.3. Let $\varphi_{0} \in V, \varphi_{1} \in H$ and denote by $\varphi$, the unique solution of (4.I) with data $\varphi_{0}, \varphi_{1}$ and $f=0$. Let us denote by $E(t)$, the energy of $\varphi$ at time $t \in[0, T]$ i.e.

$$
E(t)=\frac{1}{2}\left\{\left\|\varphi^{\prime}(t)\right\|^{2}+a(\varphi(t), \varphi(t))\right\} .
$$

Then for all $t \in[0, T]$ :

$$
E(t)=E_{0}:=\frac{1}{2}\left\{\left\|\varphi_{1}\right\|_{l l}^{2}+a\left(\varphi_{0}, \varphi_{0}\right)\right\} .
$$

Proof: In view of Theorem 4.2, it suffices to prove (4.4) for $\varphi_{0} \in D_{A}$ and $\varphi_{1} \in V$. The advantage is that the corresponding solution $\varphi$ belongs to $C\left([0, T], D_{A}\right) \cap C^{\mathrm{l}}([0, T], V) \cap C^{2}([0, T], H)$. Indeed, since $\varphi^{\prime}(t) \in V$, we deduce that

$$
\begin{aligned}
\frac{d E}{d t}(t) & =\left(\varphi^{\prime \prime}(t), \varphi^{\prime}(t)\right)_{H}+a\left(\varphi^{\prime}(t), \varphi(t)\right) \\
& =\left(\varphi^{\prime \prime}(t)+A \varphi(t), \varphi^{\prime}(t)\right)_{H}
\end{aligned}
$$

This proves that

$$
\frac{d E}{d t}(t)=0, \quad \forall t \in[0, T]
$$

and then (4.4).

Remark 4.4: Theorems 4.1 to 4.3 may be extended to the following abstract setting: $H$ and $V$ are two real Hilbert spaces such that $V$ is dense in 
$H$ and $V$ is compactly imbedded into $H ; a$ is coercive continuous bilinear form on $V$, which is symmetric.

If $u \in D_{A}$, then Theorem 3.1 shows that $u$ and $\gamma_{+} u$ admit the respective expansion (3.3) and (3.4). Unfortunately (3.3) does not imply that $u$ belongs to $H^{s}(\Omega)$, for some $s>\frac{3}{2}$. Therefore, as in paragraphs 6 and 7 of [7]; if we want to use multiplier techrics, we have to establish an identity with multiplier, which takes into account (3.3) and (3.4).

Theorem 4.5. Let us assume that $m(x)=x-x_{0}$, with $x_{0}=\left(x_{0}, \ldots, x_{0 n}\right)$ fulfilling $\mathrm{x}_{02}=0$. Then for all $u \in D_{A}$, we have:

$$
\begin{aligned}
& \int_{\Omega}\left(-\Delta_{n} u\right) m \cdot \nabla_{n} u d x \\
& +\int_{\Gamma}\left(-\Delta_{n-1}\left(\gamma_{+} u\right)+\gamma_{+} \frac{\partial u}{\partial \nu^{\ddagger}}+\gamma_{-} \frac{\partial u}{\partial \nu^{-}}\right) \gamma_{+} m \cdot \nabla_{n-1}\left(\gamma_{+} u\right) d x^{\#} \\
& =\left(1-\frac{n}{2}\right) \int_{\Omega}\left|\nabla_{n} u\right|^{2} d x+\left(1-\frac{n-1}{2}\right) \int_{\Gamma}\left|\nabla_{n-1}\left(\gamma_{+} u\right)\right|^{2} d x^{\#} \\
& -\frac{1}{2} \int_{\Gamma_{1}} m \cdot \nu\left(\gamma \frac{\partial u}{\partial \nu}\right)^{2} d \sigma-\frac{1}{2} \int_{\partial \Gamma} \gamma_{+} m \cdot \nu^{\#}\left(\gamma^{\#} \frac{\left.\frac{\partial\left(\gamma_{+} u\right)}{\frac{\partial \nu^{\#}}{2}}\right)^{2} d \sigma^{\#}}{}\right. \\
& +\pi x_{01} \int_{\Gamma_{0}} c^{2}\left(\sigma^{\sharp}\right) d \sigma^{\sharp} / 4
\end{aligned}
$$

where $c$ is the coefficient of the singularity $S_{1}$ appearing in the decomposition (3.3) of $u$.

Proof: For all $\varepsilon \in] 0,1[$, let us set

$$
\begin{aligned}
& C_{\varepsilon}=\left\{x \in \mathbf{R}^{n}: x_{1}^{2}+x_{2}^{2}<\varepsilon^{2}\right\} \\
& \Omega_{\varepsilon}=\Omega \backslash \bar{C}_{\varepsilon}, \Gamma_{\varepsilon}=\Gamma \backslash \bar{C}_{\varepsilon}, \\
& \Gamma_{\mathrm{IE}}=\partial \Omega_{\varepsilon} \cap \Gamma_{1}, \Gamma_{2 \varepsilon}=\partial \Omega_{\varepsilon} \cap \partial C_{\varepsilon} .
\end{aligned}
$$

Theorem 3.1 shows that $u \in H^{2}\left(\Omega_{c}\right)$ and $\gamma_{+} u \in H^{2}\left(\Gamma_{\varepsilon}\right)$, therefore, they fulfil the identity (3.4) of [7] respectively in $\Omega_{\varepsilon}$ and $\Gamma_{\varepsilon}$. Adding these two identities and taking into account the boundary conditions fulfilled by $u$ and $\gamma_{+} u$ and the fact that

$$
m \cdot \nu^{ \pm}=0 \quad \text { on } \Gamma,
$$


we arrive to

$$
\begin{aligned}
& \int_{\Omega_{\mathrm{c}}}\left(-\Delta_{n} u\right) m \cdot \nabla_{n} u d x \\
& +\int_{\Gamma_{\varepsilon}}\left(-\Delta_{n-1}\left(\gamma_{+} u\right)+\gamma_{+} \frac{\partial u}{\frac{\partial \nu^{+}}{2}}+\gamma_{-} \frac{\partial u}{\partial \nu^{-}}\right) \gamma_{+} m \cdot \nabla_{n-1}\left(\gamma_{+} u\right) d x^{\#} \\
& =\left(1-\frac{n}{2}\right) \int_{\Omega_{x}}\left|\nabla_{n} u\right|^{2} d x \\
& +\left(1-\frac{n-1}{2}\right) \int_{\Gamma_{\varepsilon}}\left|\nabla_{n-1}\left(\gamma_{+} u\right)\right|^{2} d x^{\#}-\frac{1}{2} \int_{\Gamma_{\mathrm{I \varepsilon}}} m \cdot \nu\left(\gamma \frac{\partial u}{\partial \nu}\right)^{2} d \sigma \\
& +\int_{\partial \Gamma_{\varepsilon}}\left\{\frac{1}{2} \gamma_{+} m \cdot \nu^{\#}\left|\nabla_{n-1}\left(\gamma_{+} u\right)\right|^{2}-\frac{\partial\left(\gamma_{+} u\right)}{\partial \nu^{\#}} \gamma_{+} m \cdot \nabla_{n-1}\left(\gamma_{+} u\right)\right\} d \sigma^{\#} \\
& +\int_{\Gamma_{2 \varepsilon}}\left\{\frac{1}{2} m \cdot \nu\left|\nabla_{n} u\right|^{2}-\frac{\partial u}{\partial \nu} m \cdot \nabla_{n} u\right\} d \sigma .
\end{aligned}
$$

We now pass to the limit as $\varepsilon$ goes to 0 . Since $u \in D_{A}$, it is clear that the lef-thand side of (4.6) tends to the left-hand side of (4.5). Moreover since $u \in H^{\prime}(\Omega), \gamma_{+} u \in H^{1}(\Gamma)$ and $u$ is regular far from the bottom of the crack, the three first terms of the right-hand side of (4.6) tend to the respective terms of the right-hand side of (4.5). So, we are reduced to study the behaviour of the integrals over $\partial \Gamma_{\varepsilon}$ and $\Gamma_{2 \varepsilon}$. Let us denote them respectively by $I_{1 \varepsilon}$ and $I_{2 \mathrm{e}}$. We shall show that

$$
\begin{gathered}
I_{1 \mathbf{c}} \rightarrow I_{1}:=-\frac{1}{2} \int_{\partial \Gamma} \gamma_{+} m \cdot \nu^{\sharp}\left(\gamma^{\#} \frac{\partial\left(\gamma_{+} u\right)}{\partial \nu^{\#}}\right)^{2} d \sigma^{\#}, \\
I_{2 \varepsilon} \rightarrow \frac{\pi x_{01}}{4} \int_{\Gamma_{0}} c^{2}\left(\sigma^{\sharp}\right) d \sigma^{\#}, \text { as } \varepsilon \rightarrow 0,
\end{gathered}
$$

which will prove (4.5).

a) Proof of (4.7): If $n=2$, we have

$$
I_{1 \varepsilon}=\frac{1}{2}\left\{m((\varepsilon, 0)) \frac{\partial(\gamma+u)}{\partial x_{1}}(\varepsilon)-m((1,0)) \frac{\partial(\gamma+u)}{\partial x_{1}}(1)\right\}
$$

Moreover, the decomposition (3.4) of $\gamma_{+} u$ implies that $\gamma_{+} u \in C^{l}(\bar{\Gamma})$ and (4.7) holds.

If $n=3$, we shall decompose the boundaries of $\Gamma_{\varepsilon}$ and $\Gamma$ as follows,

$$
\partial \Gamma_{\varepsilon}=\Gamma_{01 \varepsilon} \cup \Gamma_{02 \varepsilon}, \partial \Gamma=\Gamma_{01} \cup \Gamma_{0},
$$


where we set

$$
\begin{gathered}
\Gamma_{01 \varepsilon}=\partial \Gamma_{\varepsilon} \cap \partial \Gamma, \Gamma_{02 \varepsilon}=\left\{\left(\varepsilon, x_{3}\right):-1<x_{3}<1\right\}, \\
\Gamma_{0 \mathrm{l}}=\left\{x \in \partial \Gamma: x_{1}>0\right\} .
\end{gathered}
$$

We therefore decompose $I_{1 \varepsilon}$ (resp. $I_{1}$ ) into two integrals $I_{01 \mathrm{E}}$ and $I_{02 \mathrm{z}}$ (resp. $I_{01}$ and $I_{02}$ ) corresponding to $\Gamma_{01 \varepsilon}$ and $\Gamma_{02 \varepsilon}$ (resp. $\Gamma_{01}$ and $\Gamma_{0}$ ).

Since $\gamma_{+} u=0$ on $\Gamma_{0 \mid \mathrm{e}}$ and using Theorem 3.10, we deduce that

$$
I_{0 \mid \varepsilon} \rightarrow I_{01}, \text { as } \varepsilon \rightarrow 0 \text {. }
$$
that

For the second part, using the boundary condition $\gamma_{+} u=0$ on $\Gamma_{0}$, we see

$$
\begin{gathered}
I_{02 \varepsilon}-I_{02}=\int_{-1}^{+1}\left\{\left(\varepsilon-x_{01}\right)\left(\frac{\partial\left(\gamma_{+} u\right)}{\partial x_{1}}\left(\varepsilon, x_{3}\right)\right)^{2}\right. \\
+x_{01}\left(\frac{\partial(\gamma+u)}{\partial x_{1}}\left(0, x_{3}\right)\right)^{2}+\frac{1}{2}\left(x_{01}-\varepsilon\right)\left(\frac{\partial(\gamma+u)}{\partial x_{3}}\left(\varepsilon, x_{3}\right)\right)^{2} \\
\left.+\left(x_{3}-x_{01}\right) \frac{\partial(\gamma+u)}{\partial x_{1}}\left(\varepsilon, x_{3}\right) \frac{\partial\left(\gamma_{+} u\right)}{\partial x_{3}}\left(\varepsilon, x_{3}\right)\right\} d x_{3} .
\end{gathered}
$$

Using the next lemma, Theorem 3.10 and Lebesgue's bounded convergence theorem, we deduce that

$$
\mathrm{I}_{02 \mathrm{c}} \rightarrow I_{02} \text {, as } \varepsilon \rightarrow 0 \text {. }
$$

So the sum of (4.9) and (4.10) gives (4.7).

Lemma 4.6. Assume that $n=3$. If $u \in W^{1, p}(\Gamma)$, for some $p \geq 4 / 3$, then for every $\varepsilon \in] 0,1[$, we have

$$
\int_{-1}^{+1}\left|u^{2}\left(\varepsilon, x_{3}\right)-u^{2}\left(0, x_{3}\right)\right| d x_{3} \leq C\|u\|_{\left.W^{1, \rho}(] 0, \varepsilon[\times]-1,1\right]}\|u\|_{W^{1, \rho}(\Gamma),},
$$

where $u\left(0, x_{3}\right)$ (resp. $u\left(\varepsilon, x_{3}\right)$ ) stands for the trace of $u$ on $\Gamma_{0}$ (resp. $\Gamma_{02 \mathrm{E}}$ ). In particular,

$$
\int_{-1}^{+1}\left|u^{2}\left(\varepsilon, x_{3}\right)-u^{2}\left(0, x_{3}\right)\right| d x_{3} \rightarrow 0 \text { as } \varepsilon \rightarrow 0
$$


Proof: For $v \in \mathscr{B}(\bar{\Gamma})$, we can write

$$
v^{2}\left(\varepsilon, x_{3}\right)-v^{2}\left(0, x_{3}\right)=2 \int_{0}^{c} \frac{\partial v}{\partial x_{1}}\left(x_{1}, x_{3}\right) v\left(x_{1}, x_{3}\right) d x_{1}
$$

Integrating the absolute value of this identity and using Hölder's inequality, we get

$$
\begin{aligned}
& \int_{-1}^{+1}\left|v^{2}\left(\varepsilon, x_{3}\right)-v^{2}\left(0, x_{3}\right)\right| d x_{3} \\
& \leq 2\left\|\frac{\partial y}{\partial x_{1}}\right\|_{L^{p}(00, \varepsilon[\times]-1,1[)}\|v\|_{L^{q}(\} 0, \varepsilon[\times]-1,1[)}
\end{aligned}
$$

when $\frac{1}{p}+\frac{1}{q}=1$. But the Sobolev imbedding theorem implies that $W^{1, p}(\Gamma)$ is continuously imbedded into $L^{q}(\Gamma)$ if $1-2 / p \geq-2 / q$. This last condition is precisely equivalent to $p \geq 4 / 3$. Therefore, the inequality (4.12) can be extended to $u$ in $W^{1, p}(\Gamma)$, which implies (4.11).

b) Proof of (4.8): it is based on the following lemma:

Lemma 4.7. Let $n=3$ and $u \in H^{\prime}(\Omega)$. Then for all $\left.\varepsilon \in\right] 0, \frac{1}{2}[$, we have.

$$
\int_{-1}^{+1} \int_{0}^{2 \pi}\left|u\left(\varepsilon, \theta, x_{3}\right)\right|^{2} d \theta d x_{3} \leq C|\ln \varepsilon|\|u\|_{n^{\prime}(\Omega)}^{2},
$$

where $u\left(\varepsilon, \theta, x_{3}\right)$ stands for the trace of $u$ over $\Gamma_{2 \varepsilon}$.

Proof: Let us set

$$
\Omega^{+}=\left\{x \in \Omega: x_{2}>0\right\}, \Omega^{-}=\left\{x \in \Omega: x_{2}<0\right\} .
$$

To prove (4.13), it is sufficient to establish (4.14) and (4.15) below:

$$
\begin{aligned}
& \int_{-1}^{+1} \int_{0}^{\pi}\left|u\left(\varepsilon, \theta, x_{3}\right)\right|^{2} d \theta d x_{3} \leq C|\ln \varepsilon|\|u\|_{z^{\prime}\left(\Omega^{+}\right)^{+}}^{2}, \\
& \int_{-1}^{+1} \int_{\pi}^{2 \pi}\left|u\left(\varepsilon, \theta, x_{3}\right)\right|^{2} d \theta d x_{3} \leq C|\ln \varepsilon|\|u\|_{I I^{\prime}\left(\Omega^{-}\right)}^{2} .
\end{aligned}
$$


The reason of this splitting is that $\mathscr{P}(\bar{\Omega})$ is not dense in $H^{1}(\Omega)$, while $\mathscr{P}\left(\overline{\Omega^{+}}\right)$ (resp. $\mathscr{Q}\left(\overline{\Omega^{-}}\right)$) is dense in $H^{\prime}\left(\Omega^{+}\right)$(resp. $H^{1}\left(\Omega^{-}\right)$).

By symmetry, it suffices to prove (4.14) and only for $u \in \mathscr{Q}\left(\overline{\Omega^{+}}\right)$. For such a function, we may write

$$
\eta(\varepsilon) u\left(\varepsilon, \theta, x_{3}\right)=\int_{\varepsilon}^{1} \frac{\partial(\eta u)}{\partial r}\left(r, \theta, x_{3}\right) d r .
$$

Using Schwarz's inequality, we obtain

$$
\left|u\left(\varepsilon, \theta, x_{3}\right)\right|^{2} \leq|\ln \varepsilon| \int_{\varepsilon}^{1}\left|\frac{\partial(\eta u)}{\partial r}\right|^{2} r d r .
$$
(4.14).

Integrating this last inequality over $\left(\theta, x_{3}\right)$ in $] 0, \pi[\times]-1,1[$, we arrive to

We firstly suppose that $n=3$. Using the expansion (3.3) of $u, I_{2 \varepsilon}$ splits into a lot of terms. One of them is

$$
\frac{1}{2} \int_{-1}^{+1} \int_{0}^{2 \pi} m \cdot \nu\left|\nabla_{3} u_{0}\right|^{2} \varepsilon d \theta d x_{3}
$$

Applying Lemma 4.7 to $\frac{\partial u_{0}}{\partial x_{i}}$, for $i \in\{1,2,3\}$, which belongs to $H^{\prime}(\Omega)$, this term tends to zero.

Another term is

$$
\frac{1}{2} \int_{-1}^{+1} \int_{0}^{2 \pi} m \cdot \nu\left|\nabla_{2} c\right|^{2}\left|S_{1}\right|^{2} \varepsilon d \theta d x_{3}
$$

Using Lemma 3.9 and the definition of $S_{1}$, we see that this term is bounded by $C \varepsilon$, which tends to zero.

In the same way, using Lemmas 3.9 and 4.7 , we show that all the terms tend to zero, except the following one

$$
\frac{x_{01}}{4} \int_{-1}^{+1} \int_{0}^{2 \pi} c^{2}\left(\varepsilon, x_{3}\right) \sin ^{2}\left(\frac{\theta}{2}\right) d \theta d x_{3} .
$$

Using Lemma 4.6 , we arrive to (4.8). The proof of (4.8) is identical in dimension 2, except that no integration over $\left.x_{3} \in\right]-1,1$ [ appears. Actually, it is easier since $c$ is a constant. 
The proof of Theorem 4.5 is complete.

We are now able to establish the estimate of the energy, which is the basis of the application of HUM.

Proposition 4.8. Let $\varphi \in C\left([0, T], D_{A}\right) \cap C^{1}\left([0, T], V \cap C^{2}([0, T], H)\right.$ be a solution of

$$
\left\{\begin{array}{l}
\varphi^{\prime \prime}(t)+A \varphi(t)=0, t \in[0, T], \\
\varphi(0)=\varphi_{0}, \\
\varphi^{\prime}(0)=\varphi_{1} .
\end{array}\right.
$$

If $x_{01} \geq 0$, there exists a minimal time $T_{0}>0$ such that

$(4,17),\left(T-T_{0}\right) E_{0}$

$$
\leq C\left\{\int_{\Sigma_{1}^{+}\left(x_{0}\right)}\left(\gamma \frac{\partial \varphi}{\partial \nu}\right)^{2} d \sigma d t+\int_{\left.\Sigma_{2\left(x_{0}\right)}^{+}\right)}\left(\gamma^{\#} \frac{\partial(\gamma+\varphi)}{\partial \nu^{\sharp}}\right)^{2} d \sigma^{\#} d t\right\} .
$$

Proof: For a fixed $T$, let us set

$$
\begin{aligned}
& \left.Q_{1}=\Omega \times\right] 0, T\left[, Q_{2}=\Gamma \times\right] 0,7[, \\
& \left.\left.\Sigma_{1}=\Gamma_{1} \times\right] 0, T, \Sigma_{2}=\partial \Gamma \times\right] 0, T[.
\end{aligned}
$$

We denote

$$
I=\int_{Q_{1}} D_{1}^{2} \varphi m \cdot \nabla_{n} \varphi d x d t
$$

Integrating by parts over $] 0, T[$, we obtain

$$
I=-\int_{Q_{\mathrm{l}}} D_{\imath} \varphi m \cdot \nabla_{n}\left(D_{l} \varphi\right) d x d t+\left.\int_{\Omega} D_{r} \varphi m \cdot \nabla_{n} \varphi d x\right|_{0} ^{T} .
$$

As usual (see [12] or [7]), we show that

$$
\int_{Q_{1}} D_{t} \varphi m+\nabla_{n}\left(D_{t} \varphi\right) d x d t=-\frac{n}{2} \int_{Q_{1}}\left(D_{t} \varphi\right)^{2} d x d t .
$$

This is proven by using the Green identity in $\Omega$ in the left-hand side of (4.18) (it is allowed since $D, u \in H^{1}(\Omega)$ ) and by taking into account the fact that $m \cdot \nu^{ \pm}=0$ on $\Gamma$ and $D_{t} \varphi=0$ on $\Gamma_{1}$. 
The two previous identities lead to

$$
I=\frac{n}{2} \int_{Q_{1}}\left(D_{t} \varphi\right)^{2} d x d t+\left.\int_{\Omega} D_{1} \varphi m \cdot \nabla_{n} \varphi d x\right|_{0} ^{T} .
$$

Since $D_{1}\left(\gamma_{+} \varphi\right) \in H_{0}^{\prime}(\Gamma)$, we prove in the same way that

$$
\begin{aligned}
& \int_{Q_{2}} D_{t}^{2}\left(\gamma_{+} \varphi\right) \gamma_{+} m \cdot \nabla_{n-1}\left(\gamma_{+} \varphi\right) d x^{H} d t \\
= & \frac{n-1}{2} \int_{Q_{2}}\left(D_{t}\left(\gamma_{+} \varphi\right)\right)^{2} d x^{H} d t+\left.\int_{\Gamma} D_{t}\left(\gamma_{+} \varphi\right) \gamma_{+} m \cdot \nabla_{n-1}\left(\gamma_{+} \varphi\right) d x^{H}\right|_{0} ^{T} .
\end{aligned}
$$

Applying (4.5) to $\varphi(t)$, integrating the obtained identity over $] 0, \eta[$ and adding it with (4.19) and (4.20), we obtain :

$$
0=I(\varphi)-\frac{1}{2} \int_{\Sigma_{1}} m \cdot \nu\left(\gamma \frac{\partial \varphi}{\partial \nu}\right)^{2} d \sigma d t
$$

$$
\begin{aligned}
& -\frac{1}{2} \int_{\Sigma_{2}} \gamma_{+} m \cdot \nu^{\sharp}\left(\gamma^{\sharp} \frac{\partial(\gamma+\varphi)}{\partial \nu^{\sharp}}\right)^{2} d \sigma^{\sharp} d t+\pi x_{01} \int_{\left.\Gamma_{0} \times\right] 0, \pi} c^{2}\left(t, \sigma^{\sharp}\right) d \sigma^{\sharp} d t / 4 \\
& \quad+\left.\left[\int_{\Omega} D_{l} \varphi m \cdot \nabla_{n} \varphi d x+\int_{\Gamma} D_{l}\left(\gamma_{+} \varphi\right) \gamma_{+} m \cdot \nabla_{n-1}\left(\gamma_{+} \varphi\right) d x^{\sharp}\right]\right|_{0} ^{\gamma^{*}},
\end{aligned}
$$

where $c(t, \cdot)$ is the coefficient of the singularity $S_{1}$ appearing in the expansion (3.3) of $\varphi(t)$ and where we set

$$
\begin{aligned}
I(\varphi) & =\int_{Q_{1}}\left\{\frac{n}{2}\left(D_{1} \varphi\right)^{2}+\left(1-\frac{n}{2}\right)\left|\nabla_{n} \varphi\right|^{2}\right\} d x d t \\
& +\int_{Q_{2}}\left\{\frac{n-1}{2}\left(D_{1}\left(\gamma_{+} \varphi\right)\right)^{2}+\left(1-\frac{n-1}{2}\right)\left|\nabla_{n-1}\left(\gamma_{+} \varphi\right)\right|^{2}\right\} d x^{\#} d t .
\end{aligned}
$$

Let us now prove the following inequality

$$
I(\varphi) \geq-\frac{T E_{0}}{2}+\left.\frac{2 n-3}{4}\left[\int_{\Omega} \varphi^{\prime} \varphi d x+\int_{\Gamma}\left(\gamma_{+} \varphi\right)^{\prime} \gamma_{+} \varphi d x^{\sharp}\right]\right|_{0} ^{T} .
$$

Using the definitions of the norm in $H$ and of the bilinear form $a$, we easily check that

$$
\begin{aligned}
& I(\varphi) \geq\left(\frac{2 n-3}{4}\right) \int_{0}^{T}\left\{\left\|\varphi^{\prime}(t)\right\|_{H}^{2}-a(\varphi(t), \varphi(t))\right\} d t \\
& +\frac{1}{4} \int_{0}^{T}\left\{\left\|\varphi^{\prime}(t)\right\|_{H}^{2}+a(\varphi(t), \varphi(t))\right\} d t .
\end{aligned}
$$


Moreover, since $\varphi$ is a solution of $(4,16)$, it fulfils

$$
\left(\varphi^{\prime \prime}(t), \varphi(t)\right)_{H}+a(\varphi(t), \varphi(t))=0 \text {, a.e. } t \in[0, T] .
$$

Integrating this identity over $t \in] 0, \pi$ and integrating by parts in $t$, we arrive to

$$
\int_{0}^{T}\left\{\left\|\varphi^{\prime}(t)\right\|_{H}^{2}-a(\varphi(t), \varphi(t))\right\} d t=\left.\left(\varphi^{\prime}(t), \varphi(t)\right)_{H}\right|_{0} ^{T}
$$

The inequality (4.22) follows from (4.23), (4.24) and the identities (4.3) and $(4.4)$.

Using (4.2I), (4.22), the hypothesis $x_{01} \geq 0$, Schwarz's inequality and finally the coerciveness of the form $a$, we arrive to (4.17).

Corollary 4.9. Under the hypotheses of Proposition 4.8. If $x_{01} \geq 0$, there exists $T_{0}>0$ such that for all $T>T_{0}$, the application

$$
\begin{gathered}
\left.\left\{\varphi_{0}, \varphi_{1}\right\} \rightarrow\|\| \varphi_{0}, \varphi_{1}\right\}\|\|:=\left[\int_{\Sigma_{1}^{+}\left(x_{0}\right)}\left(\gamma \frac{\partial \varphi}{\partial \nu}\right)^{2} d \sigma d t\right. \\
\left.+\int_{\Sigma_{2}^{+}\left(x_{0}\right)}\left(\gamma^{H} \frac{\partial\left(\gamma_{+} \varphi\right)}{\partial \nu^{H}}\right)^{2} d \sigma^{H} d t\right]^{1 / 2}
\end{gathered}
$$

is a norm on $D_{A} \times V$ stronger than the norm induced by $V \times H$.

From now on, we make the following assumption

(H) We suppose that $x_{01} \geq 0$ and $T_{0}$ is the minimal time such that (4.17) holds for all $T \geq T_{0}$. We also fix $T \geq T_{0}$.

We now define $F$ as the closure of $D_{A} \times V$ for this new norm. So we have the algebraic and topological inclusions:

$$
D_{A} \times V \subset F \subset V \times H \text {. }
$$

Proposition 4.10. Under the assumption $(H)$, let $\left\{\varphi_{0}, \varphi_{1}\right\} \in F$ and $f \in L^{\prime}(0,7 ; y)$. Then there exists a unique solution $\varphi \in C([0, T], \quad) \cap$ $C^{\prime}([0, T] . H)$ of the wave equation (4.I) fulfilling

$$
\begin{aligned}
& \left\|\gamma \frac{\partial \varphi}{\partial \nu}\right\|_{L^{2}\left(\Sigma_{1}^{+}\left(x_{0}\right)\right)}+\left\|\gamma^{\#} \frac{\partial\left(\gamma_{+} \varphi\right)}{\frac{\partial \psi^{\#}}{H^{2}}}\right\|_{L^{2}\left(\Sigma_{2}^{+}\left(x_{0}\right)\right)} \\
& \leq C\left\{\left\|\left\{\varphi_{0}, \varphi_{1}\right\}\right\|+\|f\|_{L^{\prime}(0, r ; n}\right\} .
\end{aligned}
$$


Proof: We argue as in Theorem 5.6 of [7]. Using the linearity of problem (4.1) and the uniqueness of its solution, we split $\varphi=\varphi_{1}+\varphi_{2}$, where $\varphi_{1}$ corresponds to the Cauchy data (with $f=0$ ) and $\varphi_{2}$ corresponds to the righthand side $f$ (with zero Cauchy data).

The definition of $F$ insures that $\varphi_{1}$ fulfils (4.26).

Theorem 4.1 shows that $\varphi_{2} \in C\left([0, T], D_{A}\right)$ and fulfils (4.2) with $s=1$ and $\varphi_{0}=\varphi_{1}=0$. The expansion (3.3) of $\varphi_{2}(t)$ implies that $\varphi_{2}(t)$ belongs to $H^{2}$ far from the bottom of the crack. Therefore, using the trace theorem of [4], we deduce that $\gamma \frac{\partial \varphi_{2}}{\partial \nu}(t) \in L^{2}\left(\Gamma_{1}\right)$ and fulfils

$$
\left\|\gamma \frac{\partial \varphi_{2}}{\partial \nu}(t)\right\|_{L^{2}\left(\Gamma_{1}\right)} \leq C\left\|\varphi_{2}(t)\right\|_{D_{A}}
$$

where $C$ is independent of $t$.

On the other hand, Theorem 3.10 proves that $\gamma^{\sharp} \frac{\partial\left(\gamma_{+} \varphi_{2}\right)}{\partial \nu^{\prime \prime}}(t) \in L^{2}(\partial \Gamma)$ and fulfils

$$
\left\|\gamma^{\#} \frac{\partial\left(\gamma_{+} \varphi_{2}\right)}{\partial \nu^{\#}}(t)\right\|_{L^{2}(\partial \Gamma)} \leq C\left\|\varphi_{2}(t)\right\|_{D_{A}},
$$

$C$ being also independent of $t$.

Integrating the square of these two last inequalities over $] 0, T[$, we conclude that $\varphi_{2}$ also fulfils (4.26).

\section{WEAK SOLUTIONS OF THE WAVE EQUATION}

We transpose Proposition 4.10, we get

Theorem 5.1. Under the assumption ( $H)$. Then for alt $u_{0} \in H, u_{1} \in V^{*}$ $v_{1} \in L^{2}\left(\Sigma_{1}^{+}\left(x_{0}\right)\right), v_{2} \in L^{2}\left(\Sigma_{2}^{+}\left(x_{0}\right)\right)$, there exist unique $u \in L^{\infty}(0, T ; V),\left\{\psi_{1}, \psi_{0}\right\}$ $\in F^{\prime}$, which are solutions of

$$
\begin{aligned}
& \int_{0}^{T}<u(t), f(t)>d t+<\psi_{1}, \varphi_{0}>-<\psi_{0}, \varphi_{1}> \\
& =<u_{1}, \varphi(0)>-<u_{0}, \varphi^{\prime}(0)>-\int_{\Sigma_{1}^{+}\left(x_{0}\right)} v_{1} \gamma \frac{\partial \varphi}{\partial \nu} d \sigma d t \\
& -\int_{\Sigma_{2}^{+}\left(x_{0}\right)} v_{2} \gamma^{\#} \frac{\partial(\gamma+\varphi)}{\partial \nu^{\#}} d \sigma^{\prime \prime} d t,
\end{aligned}
$$


for all $f \in L !(0, T ; V),\left\{\varphi_{0},-\varphi_{1}\right\} \in F$, where $\varphi$ is the unique solution of

$$
\left\{\begin{array}{l}
\varphi \in C\left([0, T], h \cap C^{\prime}([0, T], H)\right. \\
\varphi^{\prime \prime}(t)+A \varphi(t)=f(t), t \in[0, T] \\
\varphi(T)=\varphi_{0}, \varphi^{\prime}(T)=\varphi_{1} .
\end{array}\right.
$$

Formally, the solution' $u$ of (5.1) fulfils.

$$
\left\{\begin{array}{l}
u^{\prime \prime}(t)+A u(t)=0, t \in[0, T], \\
u(0)=u_{0}, u^{\prime}(0)=u_{1}, \\
u=\left\{\begin{array}{lll}
v_{1} & \text { on } & \Sigma_{1}^{+}\left(x_{0}\right), \\
0 & \text { on } & \Sigma_{1} \backslash \Sigma_{1}^{+}\left(x_{0}\right),
\end{array}\right. \\
\gamma_{+} u=\left\{\begin{array}{lll}
v_{2} & \text { on } & \Sigma_{2}^{+}\left(x_{0}\right), \\
0 & \text { on } & \Sigma_{2} \backslash \Sigma_{2}^{+}\left(x_{0}\right),
\end{array}\right.
\end{array}\right.
$$

and the final conditions

$$
u(T)=\psi_{0}, \quad u^{\prime}(T)=\psi_{1} .
$$

This is actually the case if the data are more regular. Nevertheless, we shall give à meaning to (5.3) and we shall prove more regularity for $u$.

For these reasons, we shall say that the solution $u$ of $(5.1)$ is a weak solution of the wave equation with Cauchy data $u_{0}, u_{1}$ and boundary data $v_{1}$, $v_{2}$.

We shall need the following density result, which is proven using the particular geometry of $\Omega$ and the density of $\mathscr{Q}(\theta)$ in $L^{2}(\theta)$, when $\theta$ is any open set of $\mathbf{R}^{n}$.

Theorem 5.2. Let $v_{1} \in L^{2}\left(\Sigma_{1}^{+}\left(x_{0}\right)\right), v_{2} \in L^{2}\left(\Sigma_{2}^{+}\left(x_{0}\right)\right)$. Then there exists a sequence $v_{m} \in \mathscr{Z}\left(0, T ; C^{\infty}\left([-1,1]^{n}\right)\right)$ fulfilling

$$
\left\{\begin{array}{lll}
v_{m}=0 & \text { on } & \Sigma_{1} \backslash \Sigma_{1}^{+}\left(x_{0}\right), \\
\gamma_{+} v_{m}=0 & \text { on } & \Sigma_{2} \backslash \Sigma_{2}^{+}\left(x_{0}\right),
\end{array}\right.
$$




$$
\left\{\begin{array}{l}
v_{m}-v_{1} \text { in } L^{2}\left(\Sigma_{1}^{+}\left(x_{0}\right)\right), \\
\gamma_{+} v_{m} \rightarrow v_{2} \text { in } L^{2}\left(\Sigma_{2}^{+}\left(x_{0}\right)\right), \text { as } m \rightarrow+\infty .
\end{array}\right.
$$

Theorem 5.3. Under the assumption (H). Let $u_{0} \in V, u_{1} \in H$ and $v \in$ $\mathscr{Z}\left(0, T ; C^{\infty}\left([-1,1]^{\prime \prime}\right)\right)$ fulfilling (5.4). If $u \in L^{\infty}\left(0, T ; V^{\prime}\right)$ and $\left\{\psi_{1}, \psi_{0}\right\} \in F^{\prime}$ are the unique solutions of $(5.1)$ with data $u_{0}, u_{1}, v_{1}=\left.v\right|_{\Sigma_{1}^{+}\left(x_{0}\right)}, v_{2}=\left.\gamma_{+} v\right|_{\left.\Sigma_{2\left(x_{0}\right)}^{+}\right)}$, then

$$
u \in C([0, T], \hat{V}) \cap C^{l}([0, T], H)
$$

and fulfils (5.3), where we set

$$
\hat{V}=\left\{u \in H^{\prime}(\Omega): \gamma_{+} u=\gamma_{-} u \in H^{1}(\Gamma)\right\}
$$

Proof: We denote

$$
f_{1}=v^{\prime \prime}-\Delta_{n} v, f_{2}=\left(\gamma_{+} v\right)^{\prime \prime}-\Delta_{n-1}\left(\gamma_{+} v\right) .
$$

Since $\left(f_{1}, f_{2}\right) \in L^{2}(0, T ; H)$, by Lemma 1.3 .4 of [12], there exists a unique solution $\psi \in C([0, T], V) \cap C^{\prime}([0, T], H) \cap H^{2}\left(0, T ; V^{\prime}\right)$ of

$$
\left\{\begin{array}{l}
<\psi^{\prime \prime}(t), w>+a(\psi(t), w) \\
=-\int_{\Omega} f_{1}(t) w d x-\int_{\Gamma} f_{2}(t) \gamma_{+} w d x^{*}, \quad \text { a.e. } t \in[0, T], \forall w \in V, \\
\psi(0)=u_{0}, \psi^{\prime}(0)=u_{1} .
\end{array}\right.
$$

This means that $\psi$ is the unique solution of (4.1) with data

$$
f=-\left(f_{1}, f_{2}\right), \varphi_{0}=u_{0}, \varphi_{1}=u_{1} .
$$

We shall show that $u=v+\psi$ is the unique solution of (5.1) when

$$
\psi_{0}=u(T), \psi_{1}=u^{\prime}(T) .
$$

This will imply the desired results.

By Lemma 4.2, we are reduced to check (5.1) for $\varphi \in C\left([0, T], D_{A}\right)$ $\cap C^{\prime}([0, T], V) \cap C^{2}([0, T], H)$. Since $u \in H^{2}(0, T, V)$, integrating by parts over ]0, $T$ [ and taking into account the initial conditions fulfilled by $\varphi$ and $u$, we get

$$
\int_{0}^{T}\left\langle u(t), \varphi^{\prime \prime}+A \varphi>d t-<u(T), \varphi_{1}>+<u^{\prime}(T), \varphi_{0}\right\rangle
$$




$$
\begin{aligned}
& =<u_{1}^{\prime}, \varphi(0)>-<u_{0}, \varphi^{\prime}(0)> \\
& +\int_{0}^{T}\left\{\left\langle\psi^{\prime \prime}(t), \varphi(t)>+a(\psi(t), \varphi(t))\right.\right. \\
& \left.+<v^{\prime \prime}(t), \varphi(t)>+(v(t), A \varphi(t))_{H}\right\} d t .
\end{aligned}
$$

Applying the Green identity in $\Omega_{\varepsilon}$ and $\Gamma_{\varepsilon}$, for $\left.\varepsilon \in\right] 0,1[$, using the expansions (3.3) and (3.4) of $\varphi(t)$ and $\gamma_{+} \varphi(t)$ and letting $\varepsilon$ go to zero, we obtain :

$$
\begin{aligned}
(v, A \varphi) & =\int_{\Omega}-\Delta_{n} v \varphi d x+\int_{\Gamma}-\Delta_{n-1}\left(\gamma_{+} \nu\right) \gamma_{+} \varphi d x^{\#} \\
& -\int_{\Gamma_{1}} v_{1} \gamma \frac{\partial \varphi}{\partial \nu} d \sigma-\int_{\Gamma} v_{2} \gamma^{\#} \frac{\partial\left(\gamma_{+} \varphi\right)}{\partial \nu^{\sharp}} d \sigma^{\#} .
\end{aligned}
$$

Inserting (5.9) into (5.8) and using (5.7), we see that the right-hand side of (5.8) is equal to the right-hand side of (5.1). This is the desired identity.

Combining Theorems 5.2 and 5.3, we remark that the unique solution $u$ of (5.1) belongs to $C\left([0, T], V^{\prime}\right)$ and fulfils $u(T)=\psi_{0}$. Unfortunately, we know nothing about the regularity of its derivative. We shall obtain it by studying the first order equation associated with the wave equation (4.1).

Let us consider the real Hilbert space

$$
\mathscr{H}=V \times H,
$$

equipped with the inner product

$$
\left(\Phi_{1}, \Phi_{2}\right)_{\mathscr{H}}=a\left(\varphi_{1}, \varphi_{2}\right)+\left(\xi_{1}, \xi_{2}\right)_{H},
$$

when $\Phi_{i}=\left(\varphi_{i}, \xi_{i}\right), i=1,2$.

We introduce the linear operator $B$ defined by

$$
\begin{aligned}
& D_{B}=D_{A} \times V, \\
& \forall \Phi=(\varphi, \xi) \in D_{B}: B \Phi=(-\xi, A \varphi) .
\end{aligned}
$$

It is wellknown that $B$ is maximal monotone, then $-B$ is the infinitesimal generator of a $C_{0}$ semigroup of contractions. 
Lemma 5.4. Let $\Phi_{0} \in \mathscr{W}, F \in L^{1}(0, T ; \mathscr{Y})$. Then there exists a unique solution $\Phi \in C([0, T]$, $\not 4)$ of

$$
\left\{\begin{array}{l}
\Phi^{\prime}(t)+B \Phi(t)=F(t), t \in[0, T], \\
\Phi(0)=\Phi_{0},
\end{array}\right.
$$

which fulfils

$$
\|\Phi\|_{C([0, T] G / G)} \leq\left\|\Phi_{0}\right\|_{\mathscr{Q} /}+\|F\|_{L^{\prime}(0, T ; \mathscr{H})} .
$$

Moreover, if $\Phi_{0} \in D_{B}$ and $F \in L^{\mid}\left(0, T ; D_{B}\right)$, then $\Phi \in C\left([0, T], D_{B}\right)$ and fulfils

$$
\|\Phi\|_{C\left([0, T], D_{B}\right)} \leq\left\|\Phi_{0}\right\|_{D_{B}}+\|F\|_{L^{\prime}\left(0, T ; D_{g}\right)} .
$$

Proof: Let $S(l)$ denote the semigroup generated by $-B . \Phi$ is given by

$$
\Phi(t)=S(t) \Phi_{0}+\int_{0}^{t} S(t-s) F(s) d s .
$$

It is usually called the mild solution of (5.10) (see, for instance [15]). The estimates (5.11) and (5.12) are a direct consequence of the properties of the semigroup $S(t)$.

Actually, this lemma may be proven using Theorem 4.1 and the following matrix representation of $S(t)$ :

$$
S(t)=\left(\begin{array}{cc}
\cos t \sqrt{A} & \frac{\sin t \sqrt{A}}{\sqrt{A}} \\
-\sqrt{A} \sin t \sqrt{A} & \cos t \sqrt{A}
\end{array}\right) .
$$

In the same way, using this representation (5.14) and Proposition 4.10, we prove the

Proposition 5.5. Under the assumption (H). Let $\Phi_{0} \in F$ and $F \in$ $L^{\prime}\left(0, T, D_{B}\right)$, then the unique solution $\Phi=(\varphi, \xi) \in C([0, T], \mathscr{K})$ of $(5.10)$ fulfils

$$
\left\|\gamma \frac{\partial \varphi}{\partial \nu}\right\|_{L^{2}\left(\Sigma_{1}^{+}\left(x_{0}\right)\right)}+\left\|\gamma^{\#} \frac{\partial\left(\gamma_{+} \varphi\right)}{\partial \nu^{\#}}\right\|_{L^{2}\left(\Sigma_{2}^{+}\left(x_{0}\right)\right)} \leq C\left\{\left\|\Phi_{0}\right\|_{F}+\|F\|_{L^{1}\left(0, T, D_{B}\right)}\right\}
$$

If we transpose this Proposition 5.5, we arrive to 
$\therefore$ Theorem 5.6. Under the assumption (H). Let $U_{0} \in \mathbb{H}^{\prime}, v_{1} \in L^{2}\left(\Sigma_{1}^{+}\left(x_{0}\right)\right)$, $v_{2} \in L^{2}\left(\Sigma_{2}^{+}\left(x_{0}\right)\right)$. Then there exist unique $J \in L^{\infty}\left(0, T ; D_{B}^{\prime}\right)$ and $\psi \in F$, which are solutions of

$$
\begin{aligned}
& \text { 16) } \int_{0}^{T}<U(t), F(t)>d t+<\psi, \Phi_{0}> \\
& =<U_{0}, \Phi(0)>-\int_{\Sigma_{1}^{+}\left(x_{0}\right)} v_{1} \gamma \frac{\partial \varphi}{\partial \nu} d \sigma d t-\int_{\Sigma_{2}^{+}\left(x_{0}\right)} v_{2} \gamma^{H} \frac{\partial(\gamma+\varphi)}{\partial \nu^{H}} d \sigma^{H} d t,
\end{aligned}
$$

for all $F \in L^{\prime}\left(0, T ; D^{\prime}\right), \Phi_{0} \in F$, when $\Phi:=(\varphi, \xi) \in C([0, T], \mathcal{\&})$ is the unique solution of

$$
\left\{\begin{array}{l}
-\Phi^{\prime}(t)+B \Phi(t)=F(t), t \in[0, T] \\
\Phi(T)=\Phi_{0} .
\end{array}\right.
$$

Moreover, we have the following estimate

$$
\begin{aligned}
& \|U\|_{L^{*}\left(0, T, D_{H}\right)}+\|\psi\|_{F^{\prime}} \\
& \leq C \cdot\left\{\left\|U_{0}\right\| \xi \cdot+\left\|v_{\|}\right\|_{L^{2}\left(\Sigma_{1}\left(x_{0}\right)\right\}}+\left\|v_{2}\right\|_{L^{2}\left(\Sigma_{2}^{+}\left(x_{U}\right)\right)}\right\}
\end{aligned}
$$

It is classical that the wave equation (4.1) and the equation (5.10) are equivalent when we set $F=(0, f), \Phi_{0}=\left(\varphi_{0}, \varphi_{1}\right)$ and $\Phi=\left(\varphi, \varphi^{\prime}\right)$. Actually, we consider (5.10) since by transposition we obtain a vectorial solution $U$ of (5.16) (since $F$ has, in general, a rionzero first component). Moreover, $U$ is a formal solution of the homogeneous equation adjoint to (5.16 bis) i.e.

$$
U^{\prime}+B^{*} U=0
$$

Writting $U=(u, v)$, this identity is equivalent to

$$
\therefore, \ldots \ldots=\left\{\begin{array}{l}
u=v^{\prime} \\
y^{\prime \prime}+A v=0 .
\end{array}\right.
$$

Therefore, we may hope that $v$ is the unique solution of (5.1). Since $u=u^{\prime}$, we would obtain a regularity result for $v^{\prime}$. We now clarify these considerations in the

Theorem 5.7. Under the assumptions of Theorem 5.3. Let $u \in$ $C\left([0, T], \hat{y} \cap C^{1}([0, T], H)\right.$ be the unique solution of $(5.1)$. If we set

$$
U=\left(u^{\prime}, u\right)
$$


then $U$ is the unique solution of (5.16) with

$$
U_{0}=\left(u_{1}, u_{0}\right) \text { and } \psi=\left(u^{\prime}(T), u(T)\right) .
$$

Consequently, $u \in C\left([0, T], V^{\prime}\right) \cap C^{\prime}\left([0, T], D_{A}^{\prime}\right)$ and fulfils

$$
\begin{aligned}
& \|u\|_{C\left([0, T], V^{\prime}\right)}+\|u\|_{C^{\prime}\left([0, T], D_{A}^{\prime}\right)} \\
& \leq C\left\{\left\|u_{0}\right\|_{H}+\left\|u_{1}\right\|_{\nu^{*}}+\left\|v_{1}\right\|_{L^{2}\left(\Sigma_{1}^{+}\left(x_{0}\right)\right)}+\left\|v_{2}\right\|_{L^{2}\left(\Sigma_{2}^{+}\left(x_{0}\right)\right)}\right\} .
\end{aligned}
$$

Proof: Since $\mathscr{B}\left(0, T ; D_{B}\right)$ (resp. $\left.D_{B}\right)$ is dense in $L^{1}(0, T ; \mathscr{H})$ (resp. in $\left.\mathscr{Z}\right)$, it suffices to check that $U$ fulfils (5.16) with $F \in \mathscr{D}\left(0, T ; D_{B}\right)$ and $\Phi_{0} \in D_{B}$, the corresponding solution $\Phi$ of (5.16 bis) belonging to $C\left([0, T], D_{B}\right) \cap$

$C^{1}([0, T], \delta)$. For such a $\Phi=(\varphi, \xi)$, it is clear that $\varphi \in C^{2}([0, T], H)$. Therefore, an integration by parts over $] 0, T[$ yields

$$
\int_{0}^{T}\left\langle U(t), F(t)>d t=\int_{0}^{T}\left\langle u(t), \varphi^{\prime \prime}(t)+A \varphi(t)>d t-<u(t), \varphi^{\prime}(t)+\xi(t)>\left.\right|_{0} ^{T} .\right.\right.
$$

Since $u$ fulfils (5.1) and (5.3), the previous identity proves that $U$ fulfils (5.16) with the convention (5.19). Finally, the estimate (5.20) follows from the estimate (5.17) fulfilled by $U$.

Corollary 5.8. Under the hypotheses of Theorem 5.I the unique solution $u \in L^{\infty}\left(0, T, V^{\prime}\right)$ of (5.1) satisfies

$$
u \in C\left([0, T], V^{\prime}\right) \cap C^{l}\left([0, T], D_{A}^{\prime}\right)
$$

and the final conditions (5.3).

Proof: We use the density of $V$ in $H$, Theorems 5.2, 5.3 and 5.7 and the fact that since $V^{\prime}$ is continuously imbedded into $D_{A}^{\prime}$, the space $C\left([0, T], V^{\prime}\right)$ is continuously imbedded into $C\left([0, T], D_{A}^{\prime}\right)$.

\section{THE HILBERT UNIQUENESS METHOD}

We are now ready to give the main result of this paper.

Theorem 6.1. Under the assumption $(H)$. Then for all $u_{0} \in H$ and $u_{1} \in V^{\prime}$, there exist $v_{1} \in L^{2}\left(\Sigma_{1}^{+}\left(x_{0}\right)\right)$ and $v_{2} \in L^{2}\left(\Sigma_{2}^{+}\left(x_{0}\right)\right)$ such that the weak solution $u \in C\left([0, T], V^{\prime}\right) \cap C^{l}\left([0, T], D_{A}^{\prime}\right)$ of the wave equation (in the sense of $\left.(5.1)\right)$ 


$$
\left\{\begin{array}{l}
u^{\prime \prime}(t)+A u(t)=0, t \in[0, T], \\
u(0)=u_{0}, u^{\prime}(0)=u_{1}, \\
u=\left\{\begin{array}{lll}
v_{1} & \text { on } & \Sigma_{1}^{+}\left(x_{0}\right), \\
0 & \text { on } & \Sigma_{1} \backslash \Sigma_{1}^{+}\left(x_{0}\right),
\end{array}\right. \\
\gamma_{+} u=\left\{\begin{array}{lll}
v_{2} & \text { on } & \Sigma_{2}^{+}\left(x_{0}\right), \\
0 & \text { on } & \Sigma_{2} \backslash \Sigma_{2}^{+}\left(x_{0}\right),
\end{array}\right.
\end{array}\right.
$$

fulfils

$$
u(T)=u^{\prime}(T)=0 .
$$

Proof: By Proposition 4.10, for $\left\{\varphi_{0}, \varphi_{1}\right\} \in F$, there exists a unique solution $\varphi \in C([0, T], V) \cap C^{1}([0, T], H)$ of

$$
\left\{\begin{array}{l}
\varphi^{\prime \prime}(t)+A \varphi(t)=0, t \in[0, T] \\
\varphi(0)=\varphi_{0}, \varphi^{\prime}(0)=\varphi_{1}
\end{array}\right.
$$

which fulfils '(4.26).

Let us consider $\psi \in L^{\infty}\left(0, T ; V^{\prime}\right),\left\{\chi_{1},-\chi_{0}\right\} \in F^{\prime}$, the solutions of

$$
\begin{aligned}
& \int_{0}^{T}<\psi(t), g(t)>d t-<\chi_{1}, \eta_{0}>+<\chi_{0}, \eta_{1}> \\
& ;=-\int_{\Sigma_{1}^{+}\left(x_{0}\right)} \gamma \frac{\partial \varphi}{\partial \nu} \gamma \frac{\partial \eta}{\partial \nu} d \sigma d t \\
&-\int_{\Sigma_{2}^{+}\left(x_{0}\right)} \gamma^{\#} \frac{\partial(\gamma+\varphi)}{\partial \nu^{\#}} \gamma^{\sharp} \frac{\partial\left(\gamma_{+} \eta\right)}{\partial \nu^{\#}} d \sigma^{\#} d t,
\end{aligned}
$$

for all $g \in L^{\prime}(0, T ; V),\left\{\eta_{0}, \eta_{1}\right\} \in F$, where $\eta$ is the unique solution of

$$
\left\{\begin{array}{l}
\eta \in C\left([0, T], \eta \cap C^{\prime}([0, T], H),\right. \\
\eta^{\prime \prime}(t)+A \eta(t)=g(t), t \in[0, T], \\
\eta(0)=\eta_{0}, \eta^{\prime}(0)=\eta_{1} .
\end{array}\right.
$$


Their existence follows from Theorem 5.1 inverting the order of time. Moreover, owing to Corollary $5.8, \psi$ fulfils (5.21) and the initial conditions :

$$
\psi(0)=\chi_{0}, \psi^{\prime}(0)=\chi_{1} .
$$

We now define the operator

$$
\Lambda: F \rightarrow F^{\prime}:\left\{\varphi_{0}, \varphi_{1}\right\} \rightarrow\left\{\chi_{1},-\chi_{0}\right\} .
$$

Lemma 6.2. For all $\left\{\varphi_{0}, \varphi_{1}\right\} \in F$, we have

$$
<\Lambda\left\{\varphi_{0}, \varphi_{1}\right\},\left\{\varphi_{0}, \varphi_{1}\right\}>=\left|\left\|\left\{\varphi_{0}, \varphi_{1}\right\}\right\|\right|^{2} .
$$

Proof: Since identity (6.4) holds for all $\eta$ solution of (6.5), we may apply it with $\eta=\varphi$. This leads to

$\left.<-\chi_{1}, \varphi_{0}>+<\chi_{0}, \varphi_{1}\right\rangle=-\int_{\Sigma_{1}^{+}\left(x_{0}\right)}\left(\gamma \frac{\partial \varphi}{\partial \nu}\right)^{2} d \sigma d t-\int_{\Sigma_{2}^{+}\left(x_{0}\right)}\left(\gamma^{\#} \frac{\partial(\gamma-\varphi)}{\partial \nu^{\#}}\right)^{2} d \sigma^{\#} d t$.

This is (6.6) using the definition (4.25) of the norm in $F$.

This lemma shows that $\Lambda$ is an isomorphism from $F$ into $F^{\prime}$. Given $u_{0} \in H$, $u_{1} \in V^{\prime}$, we know that

$$
\left\{u_{1},-u_{0}\right\} \in V^{\prime} \times H \subset F^{\prime} .
$$

So there exists a unique solution $\left\{\varphi_{0}, \varphi_{1}\right\} \in F$ of

$$
\Lambda\left\{\varphi_{0}, \varphi_{1}\right\}=\left\{u_{1},-u_{0}\right\} \text {. }
$$

We build the solution $\varphi$ of (6.3) and then the solution $\psi$ of (6.4). Setting

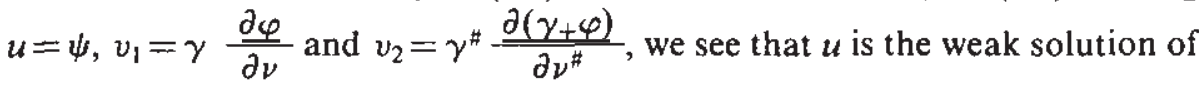
the backward wave equation

$$
\left\{\begin{array}{l}
u^{\prime \prime}(t)+A u(t)=0, t \in[0, T], \\
u(T)=u^{\prime}(T)=0, \\
u=\left\{\begin{array}{lll}
v_{t} & \text { on } & \Sigma_{1}^{+}\left(x_{0}\right), \\
0 & \text { on } & \Sigma_{1} \backslash \Sigma_{1}^{+}\left(x_{0}\right),
\end{array}\right. \\
\gamma_{+} u=\left\{\begin{array}{lll}
v_{2} & \text { on } & \Sigma_{2}^{+}\left(x_{0}\right), \\
0 & \text { on } & \Sigma_{2} \backslash \Sigma_{2}^{+}\left(x_{0}\right),
\end{array}\right.
\end{array}\right.
$$


Moreover, it fulfils

$$
u(0)=u_{0}, u^{\prime}(0)=u_{1}
$$

It remains to prove that $u$ is a weak solution of (6.1) i.e. we have to check that (since we already know that $u(T)=u^{\prime}(T)=0$ )

$$
\begin{aligned}
\int_{0}^{T}< & u(t) ; f(t)\rangle d t=\left\langle u_{1}, \varphi(0)\right\rangle-\left\langle u_{0}, \varphi^{\prime}(0)\right\rangle \\
& \quad-\int_{\Sigma_{1}^{*}\left(x_{0}\right)} v_{1} \gamma \frac{\partial \varphi}{\partial \nu} d \sigma d t-\int_{\Sigma_{2}^{+}\left(x_{n}\right)} v_{2} \gamma^{\#} \frac{\partial(\gamma+\varphi)}{\partial \nu^{\#}} d \sigma^{\#} d t,
\end{aligned}
$$

for all $f \in L^{\prime}(0,7 ; V),\left\{\varphi_{0},-\varphi_{!}\right\} \in F$, where $\varphi$ is the unique solution of (5.2).

The next lemma establishes that $\left\{\varphi(0), \varphi^{\prime}(0)\right\} \in F$. Therefore, the identity (6.4) proves (6.8) 'by setting $g=f, \eta_{0}=\varphi(0), \eta_{1}=\varphi^{\prime}(0)$ and hence $\eta=\varphi$ ).

Lemma 6.3. Let $\left\{\varphi_{0} ; \varphi_{1}\right\} \in F$ and $f \in L^{\prime}(0, T ; V)$. If $\varphi$ is the unique solution of (4.1), then

$$
\left\{\varphi(T),-\varphi^{\prime}(\dot{T})\right\} \in F
$$

Proof: By Proposition 4.10 and the reversibility of the wave equation, there exisțs a unique $\psi \in C\left([0, T], D_{A}\right) \cap C^{1}([0, T]$, $V$ of

$$
\left\{\begin{array}{l}
\psi^{\prime \prime}(t)+A \psi(t)=f(t), t \in[0, T] \\
\psi(T)=\psi^{\prime}(T)=0
\end{array}\right.
$$

fulfilling

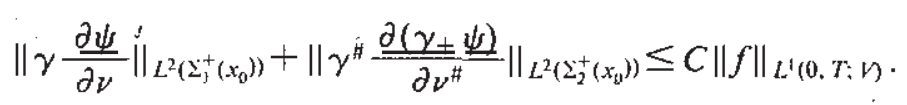

Setting $\chi=\varphi-\psi$, we deduce that $\chi \in C\left([0, T], V \cap C^{\prime}([0, T], H)\right.$ fulfils

$$
\left\{\begin{array}{l}
\chi^{\prime \prime}(t)+A \chi(t)=0, t \in[0, T] \\
\chi(T)=\varphi(T), \chi^{\prime}(T)=\varphi^{\prime}(T)
\end{array}\right.
$$

and

$$
\left\|\gamma \frac{\partial \chi}{\partial \nu^{\prime}}\right\|_{L^{2}\left(\Sigma_{*}^{+}\left(x_{0}\right)\right)}+\left\|\gamma^{\#} \frac{\partial(\gamma+\chi)}{\partial \nu^{\#}}\right\|_{L^{2}\left(\Sigma_{2}^{+}\left(x_{0}\right)\right)}<+\infty
$$

Using again the reversibility of the wave equation, this proves (6.9). 
In Theorem 6.1, we establish the exact controllability for controls with support in $\Sigma_{1}^{+}\left(x_{0}\right)$ and $\Sigma_{2}^{+}\left(x_{0}\right)$. In the physical point of view, it would be interesting to get controls having their supports only concentrated on the external boundary of $\Omega$ i.e. no control on the bottom of the crack $\Gamma_{0}$. To hit this goal, it suffices (and it is allowed) to choose $x_{0}$ such that $x_{01}=0$ in the multiplier.

\section{References}

[1] P. G. CIARLET, H. LE DRET and R. NZENGwA: Junctions between threedimensional and two-dimensional linearly elastic structures, J. Math. Pures et Appl., 68, 1989, p. 261-295.

[2] M. DAUGE: Elliptic boundary value problems on corner domains, Lecture Notes in Math., 1341, Springer-Verlag, 1988.

[3] M. DAUGE and S. NiCAISE: Oblique derivative and interface problems on polygonal domains and networks, Com. in Partial Differential Equations, 14, 1989 , p. 1147-1192.

[4] P. GRISVAR D: Théorèmes de traces relatifs à un polyèdre, C.R. Acad. Sc. Paris, t. 278, série A, 1974, p. 1581-1583.

[5] P. GRISVARD: Elliptic problems in nonsmooth domains, Monographs and studies in Math., 24, Pitman, 1985.

[6] P. GRISVARD: Edge behavior of the solution of an elliptic problem, Math. Nachriten, 132, 1987, p. 281-299.

[7] P. Grisvard: Contrôlabilité exacte des solutions de léquation des ondes en présence de singularités, J. Mąh. Pures et Appl., 68, 1989, p. 215-259.

[8] K. LEMR ABET: Problème aux limites de Ventcel dans un domaine non régulier, C.R. Acad. Sc. Paris, t. 300, Série I, 1985, p. 531-534.

[9] K. LEMRABET: Etude de divers problèmes aux limites de Ventcel d'origine physique ou mécanique dans des domaines non réguliers. Thèse, Univ. d'Alger, 1987.

[10] J.-L. Lions: Equations différentielles opérationnelles et problèmes aux limites, Grundlehren, Vol. 111, Springer-Verlag, 1961.

[11] J.-L. LIONS: Exact controllability, stabilization and perturbations for distributed systems, The J. Von Neumann Lecture, S.I.A.M. National Meeting, Boston, 1986.

[12] J.-L. Lıons: Contrôlabilité exacte, perturbations et stabilisation de systèmes distribués, tome 1, RMA 8, Masson, 1988.

[13] J.-L. LIONS and E. MAGenes: Problèmes aux limites non homogènes et applications, Vol. 1, Dunod, Paris, 1968.

[14] M. T. NIANE: Contrôlabilité exacte, de l'équation des plaques vibrantes dans, un polygone, C. R. Acad. Sc. Paris, t. 307, Série 1, 1988, p. 517-521.

[15] A. PAZY: Semigroups of linear operators and applications to partial differential equations, Applied Math. Sc., 44, Springer-Verlag, 1983.

Université de Sciences et Techniques de Lille Flandres Artois

U.F.R. de Mathérnatiques Pures et Appliquées

U.R.A C.N.R.S. 75 :

F-59655-Villeneuve d'Ascq Cedex, France 